Department of Econometrics and Business Statistics

\title{
Error-in-Variables Jump Regression Using Local Clustering
}

Yicheng Kang, Xiaodong Gong, Jiti Gao, and Peihua Qiu

July 2016 


\title{
Error-in-Variables Jump Regression Using Local Clustering
}

\author{
Yicheng Kang ${ }^{1}$, Xiaodong Gong $^{2}$, Jiti $\mathrm{Gao}^{3}$ and Peihua Qiu ${ }^{4}$ \\ JPMORGan $^{1}$, University of Canberra ${ }^{2}$, Monash University ${ }^{3}$ and University of Florida ${ }^{4}$
}

\begin{abstract}
Error-in-variables regression is widely used in econometric models. The statistical analysis becomes challenging when the regression function is discontinuous and the distribution of measurement error is unknown. In this paper, we propose a novel jump-preserving curve estimation method. A major feature of our method is that it can remove the noise effectively while preserving the jumps well, without requiring much prior knowledge about the measurement error distribution. The jump-preserving property is achieved mainly by local clustering. We show that the proposed curve estimator is statistical consistent, and it performs favorably, in comparison with an existing jump-preserving estimator. Finally, we demonstrate our method by an application to a health tax policy study in Australia.
\end{abstract}

Keywords: Clustering; Demand for private health insurance; Kernel smoothing; Local regression; Measurement errors; Price elasticity.

JEL Classification: C13, C14.

\section{Introduction}

This research is motivated by our attempt to study the impact of the medical levy surcharge tax policy on the take-up rate of private health insurance in Australia. People in Australia are liable of medical levy surcharge (which is about 1 percent of their annual taxable incomes) if they do not buy private health insurance and their annual taxable incomes are above a certain level. For example, the thresholding level for single individuals was $\$ 50,000$ per annum in the 2003-04 financial year, where the dollar sign "\$" used here and throughout the paper represents the Australian dollar. The major purpose of the medical levy surcharge 
policy was to give people more choices of health insurance and take a certain pressure off the public medical system. Both policy makers and economists are interested in studying the impact of this policy on the relationship between the private health insurance take-up rate and the annual taxable income. It was expected that this policy would generate a jump in the private health insurance take-up rate around the thresholding taxable income. This discontinuous relationship could be used to evaluate the impact of the policy. However, such relationship becomes challenging to analyze after the Australian Tax Office perturbed the income data by multiplying random numbers to them, out of privacy consideration, because the distribution that generates the random numbers was not revealed.

In the literature, jump regression analysis (cf., Qiu 2005) provides a natural framework for studying discontinuous relationship between random variables. In that framework, two approaches have been suggested for estimating a discontinuous curve. The first approach, called the indirect approach, estimates the discontinuity locations first and then considers different segments of the design interval, in which the underlying function is assumed to be continuous and can be estimated as usual. See, for example, Eubank and Speckman (1994), Gijbels et al. (1999), Gijbels and Goderniaux (2004), Kang and Qiu (2014), Kang et al. (2015), Muller (1992), Müller (2002), Qiu (1991), Qiu et al. (1991), Qiu and Kang (2015), Wu and Chu (1993), among others. The second approach, called the direct approach, estimates the regression curve directly, without first estimating the number and locations of discontinuities. Methods based on this idea include Gijbels et al. (2007), McDonald and Owen (1986), Qiu (2003), and the references therein. Most existing jump-preserving estimation methods assume that the explanatory variable does not have any measurement error involved. Error-in-variables regression models, on the other hand, allow measurement error in the explanatory variables. But most of them assume that the regression function is smooth and that the measurement error distribution is known or it can be estimated reasonably well beforehand (cf., Carroll et al. 1999, 2012, Comte and Taupin 2007, Cook and Stefanski 1994, Delaigle and Meister 2007, Fan and Masry 1992, Fan and Truong 1993, Hall and Meister 2007, Staudenmayer and Ruppert 2004, Stefanski 2000, Stefanski and Cook 1995, and Taupin 2001). 
In this paper, we propose a jump-preserving curve estimation method for discontinuous Error-in-variables regression models. The proposed method is a direct approach without explicitly detecting jumps first and thus it is easy to use. Another feature of our method is that it does not require the measurement error distribution to be specified beforehand, making it applicable to many real problems. The remainder of this article is organized as follows. In Section 2, our proposed method is described in detail. In Section 3, some asymptotic properties of the proposed estimator are discussed. In Section 4, the numerical performance is evaluated by simulated examples. In Section 5, the proposed method is applied to the private health insurance data. Several remarks conclude the article in Section 6. Some technical details are provided in a supplementary file.

\section{Proposed Methodology}

Let $\left\{\left(W_{i}, Y_{i}\right): i=1, \ldots, n\right\}$ be independent and identically distributed observations from the model described below.

$$
\begin{aligned}
Y_{i} & =g\left(X_{i}\right)+\varepsilon_{i}, \\
W_{i} & =X_{i}+\sigma_{n} U_{i},
\end{aligned}
$$

where $i=1, \ldots, n, g$ is the unknown regression function with possible discontinuities, $Y_{i}$ is the $i$ th observation of the response variable, $X_{i}$ is the $i$ th observation of the unobservable explanatory variable, $\varepsilon_{i}$ 's are independent and identically distributed random errors with mean 0 and unknown variance $\tau^{2}>0, W_{i}$ is the observed value of $X_{i}$ with a measurement error, $\sigma_{n}>0$ denotes the standard deviation of the measurement error in $X_{i}$, and $U_{i}$ is the standardized measurement error with mean 0 and variance 1 . It is also assumed that $U_{i}$ 's are independent and identically distributed, $U_{i}$ is independent of both $X_{i}$ and $Y_{i}$, the distribution of $U_{i}$, denoted as $f_{U}$, and the distribution of $X_{i}$, denoted as $f_{X}$, are both unknown. Without loss of generality, assume that the design interval is $[0,1]$. Our major goal is to estimate $g(x)$ from the observed data.

Our idea of estimating a regression function with possible jump points of unknown jump 
locations is that each point in the design interval is a potential jump point and thus the estimation method should adapt at each point to a possible discontinuity. Next, we describe the proposed method in detail. For any given point $x \in\left[h_{n}, 1-h_{n}\right]$, where $h_{n} \in(0,1 / 2)$ is a bandwidth parameter, consider a small neighborhood of $x$ defined by

$$
N\left(x ; h_{n}\right)=\left\{z \in(0,1):|z-x| \leq h_{n}\right\}
$$

and the following local linear kernel (LLK) smoothing procedure:

$$
\min _{a, b} \sum_{N\left(x ; h_{n}\right)}\left[Y_{i}-a-b\left(W_{i}-x\right)\right]^{2} K\left(\frac{W_{i}-x}{h_{n}}\right),
$$

where $K$ is a density kernel function with support $[-1,1]$. Let $\left(\widehat{a}_{n}(x), \widehat{b}_{n}(x)\right)$ be the solution to $(a, b)$ in (3). Then, the weighted residual mean squares (WRMS) at $x$ is defined by

$$
\operatorname{WRMS}_{n}(x)=\frac{\sum_{N\left(x ; h_{n}\right)}\left[Y_{i}-\widehat{a}_{n}(x)-\widehat{b}_{n}(x)\left(W_{i}-x\right)\right]^{2} K\left(\frac{W_{i}-x}{h_{n}}\right)}{\sum_{N\left(x ; h_{n}\right)} K\left(\frac{W_{i}-x}{h_{n}}\right)} .
$$

If $x$ is a jump point, then the jump structure of the regression function would be dominant even when there is a measurement error involved. This fact is illustrated in Fig. 1. By this observation, if $x$ is near a jump point, there should be a significant evidence of lack-of-fit of the LLK smoothing procedure (3). In other words, $\mathrm{WRMS}_{n}(x)$ would be relatively large. Thus, if the following is true:

$$
\operatorname{WRMS}_{n}(x)>u_{n},
$$

where $u_{n}$ is a threshold value, then $x$ is likely to be close to a jump point, and we cannot use all observations near $x$ to estimate $g(x)$ because it would blur the jump otherwise. When there is no measurement error in $X$, the one-sided estimators can estimate $g(x)$ reasonably well (cf., Qiu 2003). In the case when $X$ has measurement error involved, such estimators are unavailable because $X_{i}$ 's are no longer observable. It may be problematic if we simply replace $X_{i}$ 's by $W_{i}$ 's for constructing a one-sided estimator because we do not know whether a specific value $X_{i}$ is located on the right (or left) side of $x$ when its observed value $W_{i}$ is on the right (or left) side of $x$, due to the measurement error. To overcome this difficulty, 


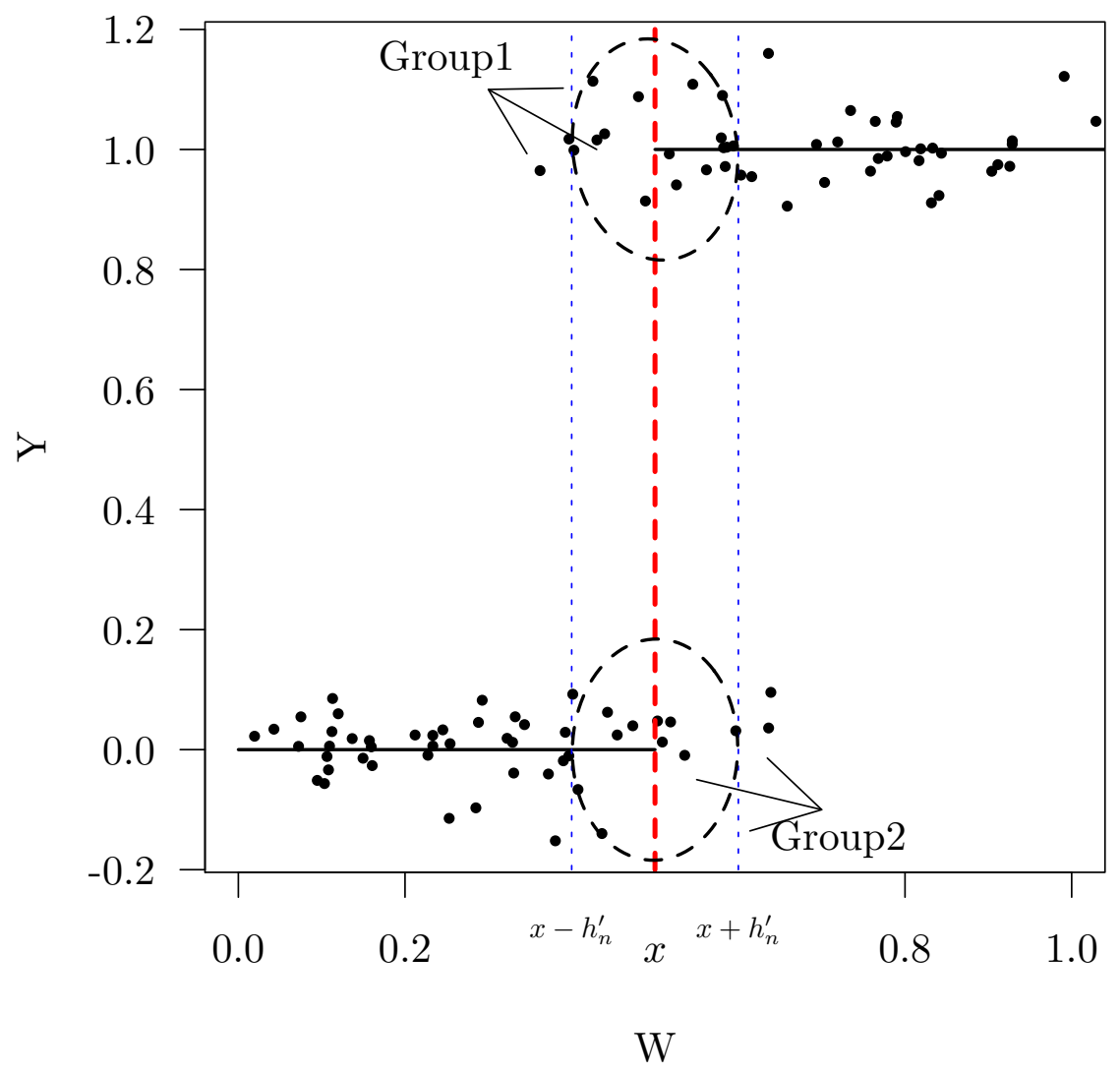

Figure 1: The solid line denotes the regression function $g(\cdot)$ that has a jump at $x=0.5$ (marked by the vertical dashed line). The dark points denote observations of $(W, Y)$ where $W$ is the observed value of $X$ with measurement error involved. It can be seen that the jump structure of $g(\cdot)$ is quite visible among observations in $N\left(x ; h_{n}^{\prime}\right)$ (i.e., those fall between the two vertical dotted lines) even in the presence of measurement error. 
we can make use of the fact that the jump structure of $g(\cdot)$ is still quite visible even in the presence of measurement error (cf., Fig. 1). We suggest classifying all observations in the neighborhood $N\left(x ; h_{n}^{\prime}\right)$ of $x$ into two significantly separated groups, (i.e., Group 1 versus Group 2 in Fig. 1), where the bandwidth parameter $h_{n}^{\prime}$ could be different from $h_{n}$. Then, we can estimate $g(x)$ using the observations in one group. As long as the observations are properly clustered, the jump should be preserved well.

Next, we describe our clustering procedure mentioned above. An ideal classification would put observations whose unobservable $X$ values are on the same side of the jump location into a same group. So, a classification should be reasonable when certain separation measure reaches the maximum. Intuitively, if the two groups of observations are well separated, the within-group variability would be small and the between-group variability would be large. Consequently, the ratio of between-group variability and within-group variability would be large. Therefore, we can use this ratio as a separation measure of the two groups. Specifically, let $G\left(x ; h_{n}^{\prime}\right)=\left\{\left(W_{i}, Y_{i}\right): W_{i} \in N\left(x ; h_{n}^{\prime}\right)\right\}, G_{l}\left(x ; h_{n}^{\prime}\right)$ and $G_{r}\left(x ; h_{n}^{\prime}\right)$ be a partition of $G\left(x ; h_{n}^{\prime}\right)$ (i.e., $G\left(x ; h_{n}^{\prime}\right)=G_{l}\left(x ; h_{n}^{\prime}\right) \cup G_{r}\left(x ; h_{n}^{\prime}\right)$ and $\left.G_{l}\left(x ; h_{n}^{\prime}\right) \cap G_{r}\left(x ; h_{n}^{\prime}\right)=\emptyset\right)$, and

$$
\begin{aligned}
& \bar{W}_{l}=\frac{1}{\left|G_{l}\left(x ; h_{n}^{\prime}\right)\right|} \sum_{\left(W_{i}, Y_{i}\right) \in G_{l}\left(x ; h_{n}^{\prime}\right)} W_{i}, \quad \bar{Y}_{l}=\frac{1}{\left|G_{l}\left(x ; h_{n}^{\prime}\right)\right|} \sum_{\left(W_{i}, Y_{i}\right) \in G_{l}\left(x ; h_{n}^{\prime}\right)} Y_{i}, \\
& \bar{W}_{r}=\frac{1}{\left|G_{r}\left(x ; h_{n}^{\prime}\right)\right|} \sum_{\left(W_{i}, Y_{i}\right) \in G_{r}\left(x ; h_{n}^{\prime}\right)} W_{i}, \quad \bar{Y}_{r}=\frac{1}{\left|G_{r}\left(x ; h_{n}^{\prime}\right)\right|} \sum_{\left(W_{i}, Y_{i}\right) \in G_{r}\left(x ; h_{n}^{\prime}\right)} Y_{i},
\end{aligned}
$$

where $|A|$ denotes the number of elements in the pointset $A$. Next, we consider the following LLK smoothing procedures:

$$
\min _{a, b} \sum_{\left(W_{i}, Y_{i}\right) \in G_{l}\left(x ; h_{n}^{\prime}\right)}\left[Y_{i}-a-b\left(W_{i}-x\right)\right]^{2} K\left(\frac{W_{i}-x}{h_{n}^{\prime}}\right),
$$

and

$$
\min _{a, b} \sum_{\left(W_{i}, Y_{i}\right) \in G_{r}\left(x ; h_{n}^{\prime}\right)}\left[Y_{i}-a-b\left(W_{i}-x\right)\right]^{2} K\left(\frac{W_{i}-x}{h_{n}^{\prime}}\right) .
$$

And the WRMS's defined in (4) can be computed after $N\left(x ; h_{n}\right)$ is replaced by $G_{l}\left(x ; h_{n}^{\prime}\right)$ and $G_{r}\left(x ; h_{n}^{\prime}\right)$, respectively. They are denoted as $\operatorname{WRMS}_{l}\left(x ; h_{n}^{\prime}\right)$ and $\operatorname{WRMS}_{r}\left(x ; h_{n}^{\prime}\right)$. Then, 
we define the following separation measure:

$$
T\left(G_{l}\left(x ; h_{n}^{\prime}\right), G_{r}\left(x ; h_{n}^{\prime}\right)\right)=\frac{\left(\bar{W}_{l}-\bar{W}_{r}\right)^{2}+\left(\bar{Y}_{l}-\bar{Y}_{r}\right)^{2}}{\operatorname{WRMS}_{l}\left(x ; h_{n}^{\prime}\right)+\operatorname{WRMS}_{r}\left(x ; h_{n}^{\prime}\right)} .
$$

It can be seen that the numerator in (8) represents between-group variability and the denominator represents within-group variability. Let $G_{l}^{*}\left(x ; h_{n}^{\prime}\right)$ and $G_{r}^{*}\left(x ; h_{n}^{\prime}\right)$ denote the partition that maximizes (8). In practice, solving the optimization in (8) by exhaustive search would be too time-consuming (cf., Everitt et al. 2011, Chapter 5). The more efficient algorithm proposed in Hartigan and Wong (1979) is well received in the literature. We adopt that algorithm in this paper. Let $\widehat{a}_{l, n}(x)$ and $\widehat{a}_{r, n}(x)$ denote the solution to $a$ in (6) and (7), respectively. If $\left|G_{l}^{*}\left(x ; h_{n}^{\prime}\right)\right|>\left|G_{r}^{*}\left(x ; h_{n}^{\prime}\right)\right|$, then it is more likely for $x$ to be on the same side of the jump point as the $X$ values of those observations in $G_{l}^{*}\left(x ; h_{n}^{\prime}\right)$. So, our proposed estimator of $g(x)$ is

$$
\widehat{g}_{n}(x)= \begin{cases}\widehat{a}_{l, n}(x), & \text { if }\left|G_{l}^{*}\left(x ; h_{n}^{\prime}\right)\right|>\left|G_{r}^{*}\left(x ; h_{n}^{\prime}\right)\right|, \\ \widehat{a}_{r, n}\left(x^{\prime}\right), & \text { otherwise. }\end{cases}
$$

The proposed jump-preserving curve estimation procedure is summarized as follows.

\section{Jump-preserving Curve Estimation Procedure}

Step 1: For any given $x$, compute its WRMS by (4).

Step 2: If (5) is true, go to Step 3. Otherwise, estimate $g(x)$ by $\widehat{a}_{n}(x)$, the solution to $a$ in $(3)$.

Step 3: Cluster the observations in $G\left(x ; h_{n}^{\prime}\right)$ by maximizing $(8)$, then compute $\widehat{g}_{n}(x)$ by (9).

In the proposed estimation procedure (3)-(9), there are three parameters, $h_{n}, h_{n}^{\prime}$ and $u_{n}$, to choose. $h_{n}$ is used for two purposes: to flag possible jump points in (5) and to estimate the curve in continuity regions. The purpose that $h_{n}$ serves in our procedure is similar to what the bandwidth parameter does in the conventional kernel smoothing. Thus, we suggest to select $h_{n}$ to minimize the following leave-one-out cross validation score:

$$
\min _{h_{n}} \sum_{i=1}^{n}\left[Y_{i}-\widehat{a}_{n}^{(-i)}\left(W_{i}\right)\right]^{2}
$$


where $\widehat{a}_{n}^{(-i)}(\cdot)$ denotes the estimate $\widehat{a}_{n}(\cdot)$ when the $i$ th observation $\left(W_{i}, Y_{i}\right)$ is omitted.

Next, we discuss the selection of $h_{n}^{\prime}$ and $u_{n}$. In simulation studies, the true regression function $g$ could be known. Then, once $h_{n}$ is selected, $\left(h_{n}^{\prime}, u_{n}\right)$ can be chosen to be the pair that minimizes the Mean Square Error (MSE), defined as

$$
\operatorname{MSE}\left(\widehat{g}, g ; h_{n}^{\prime}, u_{n}\right)=\frac{1}{n} \sum_{i=1}^{n}\left[\widehat{g}\left(x_{i}\right)-g\left(x_{i}\right)\right]^{2},
$$

where $\left\{x_{1}, \ldots, x_{n}\right\}$ are equally spaced values on $[0,1]$. In practice, $g$ is usually unknown. In such cases, we suggest the following bootstrap selection procedure:

- For a given bandwidth value $h_{n}^{\prime}>0$ and threshold value $u_{n}>0$, apply the proposed estimation procedure (3)-(9) to the original dataset $\left\{\left(W_{1}, Y_{1}\right), \ldots,\left(W_{n}, Y_{n}\right)\right\}$, and obtain an estimator of $g$, denoted as $\widehat{g}\left(\cdot ; h_{n}^{\prime}, u_{n}\right)$.

- Draw with replacement $n$ times from the original dataset to obtain the first bootstrap sample, denoted as $\left\{\left(\widetilde{W}_{1}^{(1)}, \widetilde{Y}_{1}^{(1)}\right), \ldots,\left(\widetilde{W}_{n}^{(1)}, \widetilde{Y}_{n}^{(1)}\right)\right\}$.

- Apply the proposed estimation procedure (3)-(9) to the first bootstrap sample, and obtain the first bootstrap estimator of $g$, denoted as $\widetilde{g}^{(1)}\left(\cdot ; h_{n}^{\prime}, u_{n}\right)$.

- Repeat the previous two steps $B$ times and obtain $B$ bootstrap estimators of $g$ : $\left\{\widetilde{g}^{(1)}\left(\cdot ; h_{n}^{\prime}, u_{n}\right), \ldots, \widetilde{g}^{(B)}\left(\cdot ; h_{n}^{\prime}, u_{n}\right)\right\}$.

- Then, the bandwidth $h_{n}^{\prime}$ and the threshold $u_{n}$ are chosen to be the minimizer of

$$
\min _{h_{n}^{\prime}, u_{n}} \frac{1}{B} \sum_{k=1}^{B} \frac{1}{n} \sum_{i=1}^{n}\left[\widetilde{g}^{(k)}\left(x_{i} ; h_{n}^{\prime}, u_{n}\right)-\widehat{g}\left(x_{i} ; h_{n}^{\prime}, u_{n}\right)\right]^{2}
$$

\section{Asymptotic Properties}

In this section, we discuss some asymptotic properties of the proposed estimation procedure (3)-(9). To this end, we have the theorem below.

Theorem 1. Suppose that the following conditions hold: $\left\{\left(W_{1}, Y_{1}\right), \ldots,\left(W_{n}, Y_{n}\right)\right\}$ are independent and identically distributed observations from models (1) and (2). $g(\cdot)$ is a bounded, piecewise continuous function defined on $[0,1]$ with finitely many jump points in $[0,1]$; at each jump point, $g(\cdot)$ has finite one-sided limit; its first-order derivative, $g^{\prime}(\cdot)$, is also a bounded 
function and is continuous on $[0,1]$ except on those jump points; at each jump point, $g^{\prime}(\cdot)$ also has finite one-sided limit. Denote the set of all the jump points by S. The support of $f_{X}$ is $[0,1] ; f_{X}$ is uniformly continuous, bounded, and positive on $(0,1)$ and has bounded derivatives on $(0,1) . f_{U}$ is continuous on its support, symmetric about 0 with $f_{U}(0)>0$ and satisfies the conditions that $\int_{-\infty}^{\infty} u f_{U}(u) d u=0$ and $\int_{-\infty}^{\infty} u^{2} f_{U}(u) d u=1 . E\left|\varepsilon_{1}\right|^{4}<\infty$. $h_{n}=o(1), 1 /\left(n^{1 / 3} h_{n}\right)=o(1), h_{n}^{\prime}=o(1), \sigma_{n}^{2} / h_{n}^{\prime}=o(1)$, and $1 /\left(n^{1 / 3} h_{n}^{\prime}\right)=o(1)$ The kernel function $K$ is a Lipschitz-1 continuous density function with support $[-1,1]$ and is symmetric about $0 . u_{n}=\tau^{2}+\delta_{n}$, where $\delta_{n}$ is sequence of positive numbers such that $\delta_{n}=o(1)$ and that $\left[h_{n}^{2}+\sigma_{n}^{2}+(\log n)^{1+\gamma} /\left(n h_{n}\right)^{\beta}\right] / \delta_{n}=o(1)$ for some $\gamma>0$ and some $\beta \in(0,1 / 4)$. Then, we have, with probability 1 ,

$$
\widehat{g}_{n}(x)-g(x)= \begin{cases}O\left(h_{n}^{2}+\sigma_{n}^{2}+(\log n)^{1+\gamma} /\left(n h_{n}\right)^{\beta}\right), & \text { if } d_{E}(x, S)>h_{n}, \\ O\left(h_{n}^{2}+\sigma_{n}^{2}+(\log n)^{1+\gamma} /\left(n h_{n}^{\prime}\right)^{\beta}\right), & \text { otherwise, }\end{cases}
$$

where $S$ is the set of all true jump points and $d_{E}(x, S)=\min _{x_{s} \in S}\left|x-x_{s}\right|$.

The Theorem 1 shows that the proposed estimation procedure (3)-(9) estimates $g(\cdot)$ consistently under some regularity conditions. Its proof is given in a supplementary file.

Remark 1 Theorem 1 requires that the measurement error standard deviation $\sigma_{n}$ tends to 0 when the sample size increases. In the literature, it has been pointed out that this condition is needed for consistently estimating the regression function when its observations have measurement errors involved and when little prior information about the measurement error distribution is available (cf., Delaigle 2008).

Remarks 2 The rate of convergence of $\sigma_{n}$ to 0 does not need to be comparable to that of $h_{n}$ for our proposed method to flag all jump points correctly (see the supplementary file for details). However, the condition $\sigma_{n}^{2} / h_{n}^{\prime}=o(1)$ is required for classifying a jump point into the correct cluster. This is a weaker condition than the one $\sigma_{n} / h_{n}^{\prime}=o(1)$ which would be required by existing jump regression methods (e.g., Gijbels et al. 2007, Qiu 2003) to ensure consistency. 


\section{Numerical Studies}

\subsection{Numerical performance of the proposed methodology}

In this subsection, the performance of the proposed estimation procedure is evaluated using the following two true regression functions:

$$
\begin{aligned}
& g_{1}(x)=\left(3 x^{2}+0.53\right) \mathbb{1}_{\{0.3 \leq x<0.7\}}+\left(2 x^{2}+2.22\right) \mathbb{1}_{\{0.7 \leq x \leq 1\}}, \\
& g_{2}(x)=\cos (4 \pi(0.5-x)) \mathbb{1}_{\{0 \leq x<0.5\}}-\cos \left((4 \pi(x-0.5)) \mathbb{1}_{\{0.5 \leq x \leq 1\}},\right.
\end{aligned}
$$

where $\mathbb{1}_{\{\cdot\}}$ is an indicator function taking the value of 1 if the argument in the brace is true and 0 otherwise. $g_{1}$ and $g_{2}$ are graphed in Fig. 2(a) and Fig. 2(c), respectively. It can be seen that $g_{1}$ has two jump points. One is at $x=0.3$ with jump size 0.8 and the other one is at $x=0.7$ with jump size 0.8 . $g_{2}$ has a single jump of size 2 at $x=0.5$. For each regression function, we consider cases when the sample size $n$ equals 500 or $1000, f_{X} \sim \operatorname{Unif}[0,1]$, and $f_{U}$ is either a Normal, a Laplace, or a Uniform distribution with $\mathrm{E}(U)=0$ and $\operatorname{var}(U)=1$. $\tau$ and $\sigma_{n}$ are fixed at 0.1 and 0.05 , respectively. In each combination of $g, n$, and $f_{U}$, the simulation is repeated 200 times. For each given bandwidth $h_{n}^{\prime}$ and threshold value $u_{n}$, 200 values of $\operatorname{MSE}\left(\widehat{g}, g ; h_{n}^{\prime}, u_{n}\right)$ defined in (10) are computed. Their average is called the Average Mean Square Error (AMSE) and is denoted as $\operatorname{AMSE}\left(h_{n}^{\prime}, u_{n}\right)$. The minimizer of $\operatorname{AMSE}\left(h_{n}^{\prime}, u_{n}\right)$ is called the optimal bandwidth and the optimal threshold, and is denoted as $h_{o p t}^{\prime}$ and $u_{o p t}$, respectively. We also compute the bandwidth value and threshold value using the proposed bootstrap selection procedure. Such bandwidth and threshold are called the bootstrap bandwidth and the bootstrap threshold, denoted as $h_{b t}^{\prime}$ and $u_{b t}$, respectively. Throughout this section, if there is no further specification, the bootstrap sample size $B$ is chosen to be 200, and $K$ used in (3), (4), (6), and (7) is chosen to be the Epanechnikov kernel function (i.e., $\left.K(x)=0.75\left(1-x^{2}\right) \mathbb{1}_{\{|x| \leq 1\}}\right)$. The values of $h_{o p t}^{\prime}, u_{o p t}, h_{b t}^{\prime}$, $u_{b t}$, and $\operatorname{AMSE}\left(h_{\text {opt }}^{\prime}, u_{\text {opt }}\right)$ are presented in Table 1.

From the table, it can be seen that (i) the performance of the proposed estimation procedure improves as the sample size $n$ increases, and (ii) the bootstrap selection procedure chooses parameters close to the optimal ones. 
Table 1: Numerical summary of two simulation examples based on 200 replicated simulations

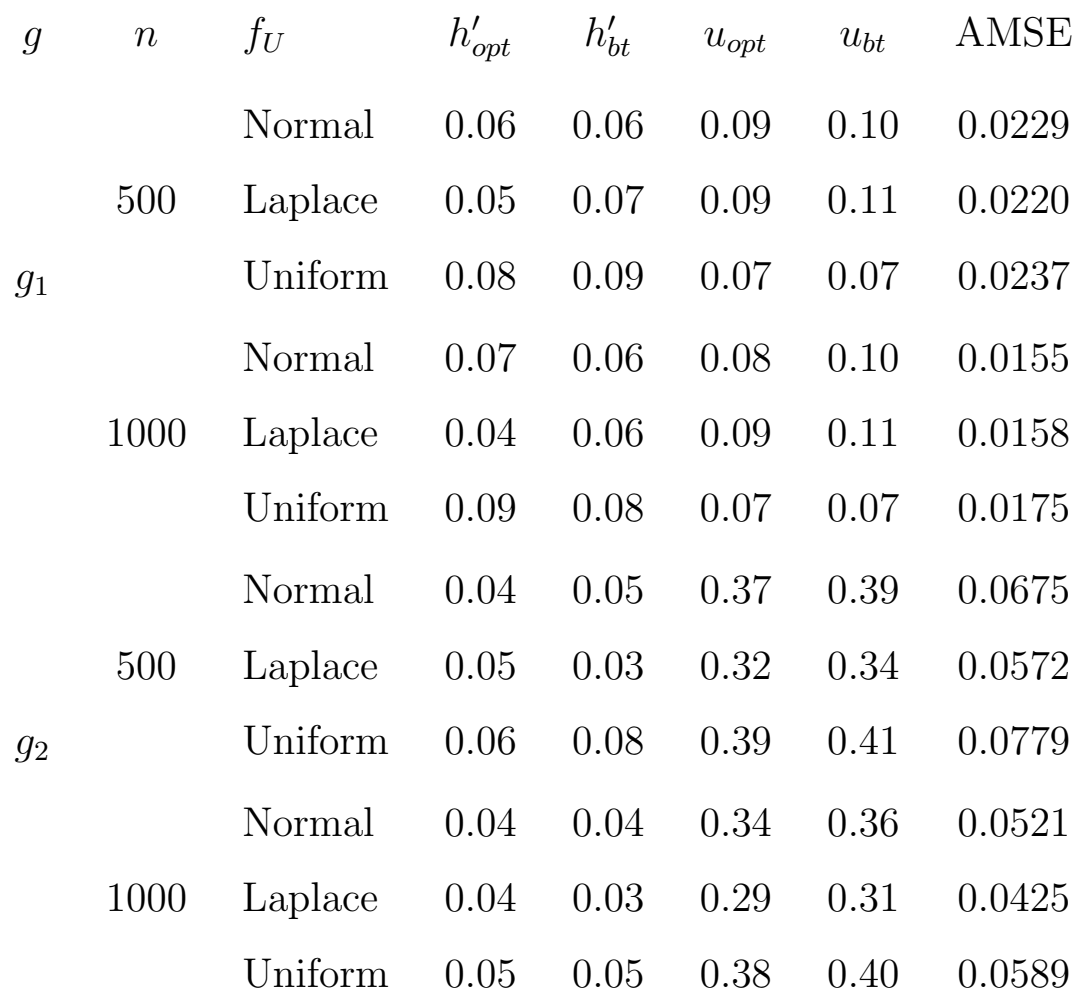


Next, in the case when $n=500$ and $f_{U}$ is Normal, the realizations of $\left\{\left(W_{i}, Y_{i}\right): i=\right.$ $1, \ldots, n\}$ when $g$ is $g_{1}$ and $g_{2}$ and their corresponding estimates are shown in Fig. 2(b) and Fig. 2(d), respectively. It can be seen that our curve estimation procedure preserves the jumps well in the presence of measurement error. It also can be seen that there were little kinks near the jump points in the estimated curves. This is because when $\operatorname{WRMS}_{n}(x)$ approaches to but does not exceed the threshold $u_{n}, \widehat{g}_{n}(x)=\widehat{a}_{n}(x)$ which uses observations from both sides of the jump point. As $x$ gets closer to the jump point, once $\operatorname{WRMS}_{n}(x)$ exceeds $u_{n}$, the clustering procedure kicks in and $\widehat{g}_{n}(x)$ will use the observations from one cluster only. Thus, the slight kinky behavior is mainly caused by the hard thresholding used in the proposed procedure.

\subsection{Comparison to piecewise-linear kernel estimator}

The piecewise-linear kernel estimator proposed by Qiu (2003) is a direct curve estimation method that preserves jumps well when there is no measurement error involved. In this subsection, we compare our proposed procedure with Qiu (2003) in an artificial example. The proposed procedure is denoted as NEW and the procedure in Qiu (2003) is denoted as PLKE. Assume that the regression function is

$$
g(x)= \begin{cases}-\frac{25}{9}(x-0.6)^{2}, & \text { if } x \in[0,0.6), \\ 4(x-0.6)^{3}+0.5, & \text { if } x \in[0.6,1.0] .\end{cases}
$$

It can be seen that $g(x)$ is a piecewise polynomial with a jump size 0.5 at $x=0.6$, as plotted in Fig. 3(a) (the solid line). In this numerical comparison, we choose the sample size $n$ to be $500, \tau$ to be $0.05, f_{U}$ to be $N\left(0,0.1^{2}\right)$, and $f_{X}$ to be either $\operatorname{Unif}[0,1]$, $\operatorname{Beta}(2,2)$, $\operatorname{Beta}(3,2)$ or $\operatorname{Beta}(2,3)$. In each case, the simulation is repeated 200 times, the optimal parameters (i.e., the bandwidth-threshold pair for NEW and the bandwidth parameter for PLKE) are selected based on the AMSE from 200 replicated simulations. The AMSEs and their standard deviations (denoted as SDAMSE) are computed. These results are presented in Table 2. From Table 2, it can be seen that the proposed procedure outperforms PLKE, across all difference choices of $f_{X}$. 


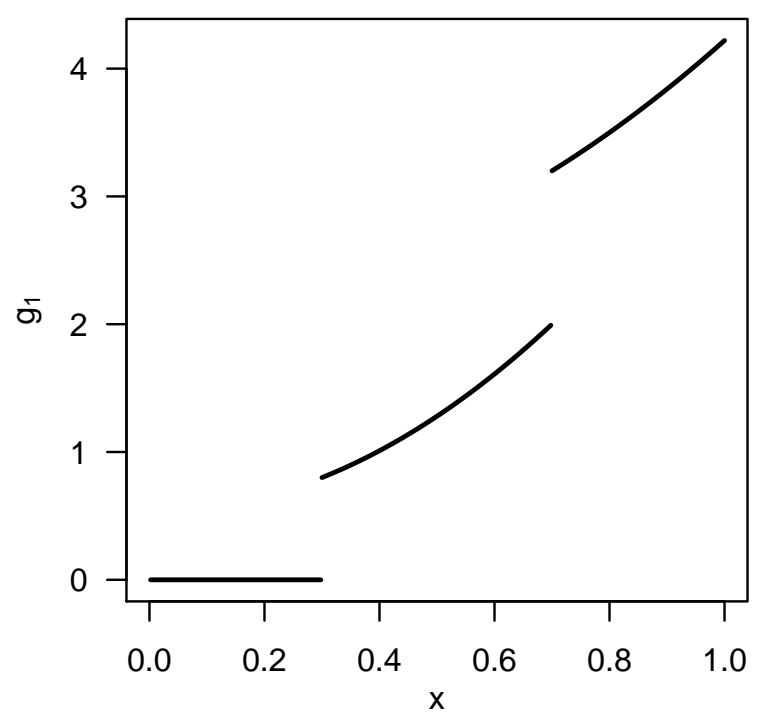

(a)

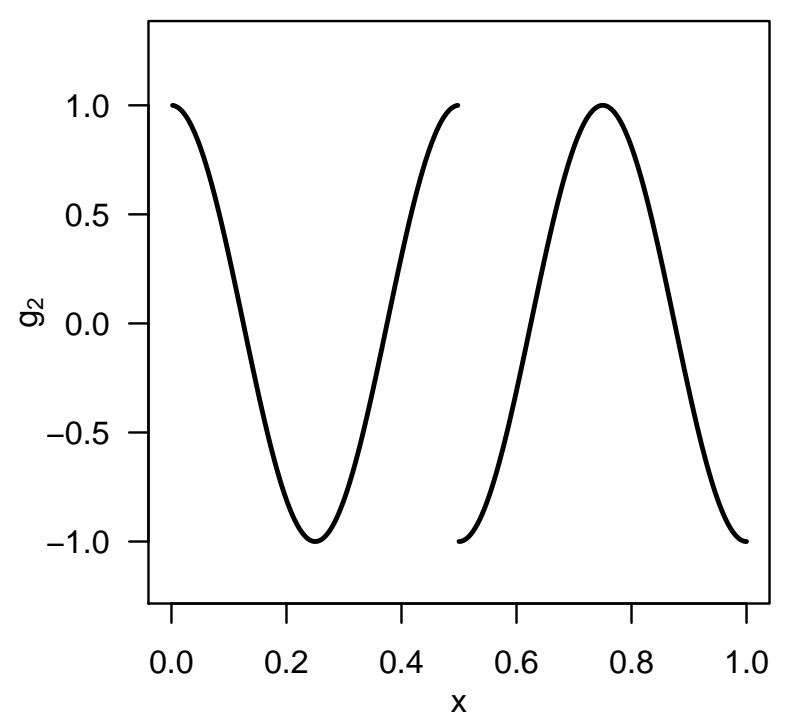

(c)

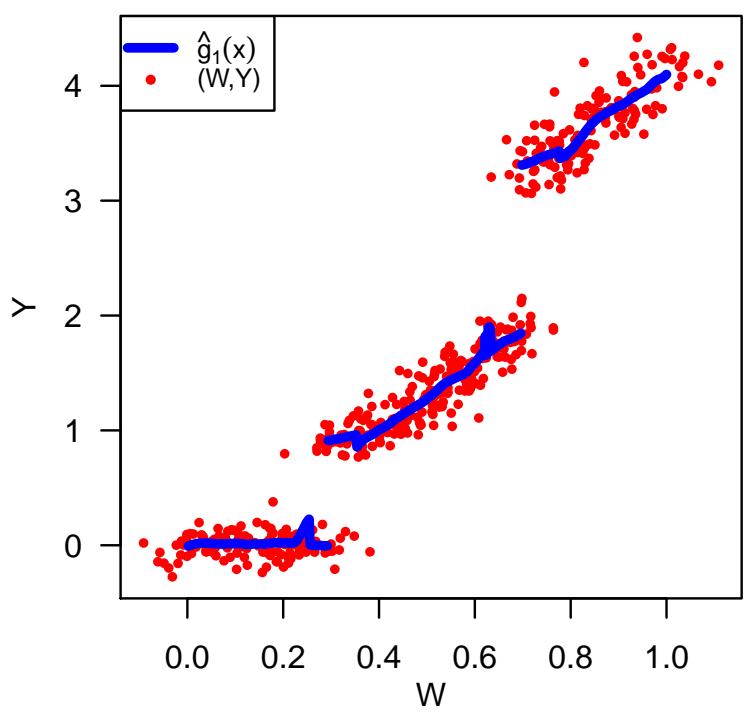

(b)

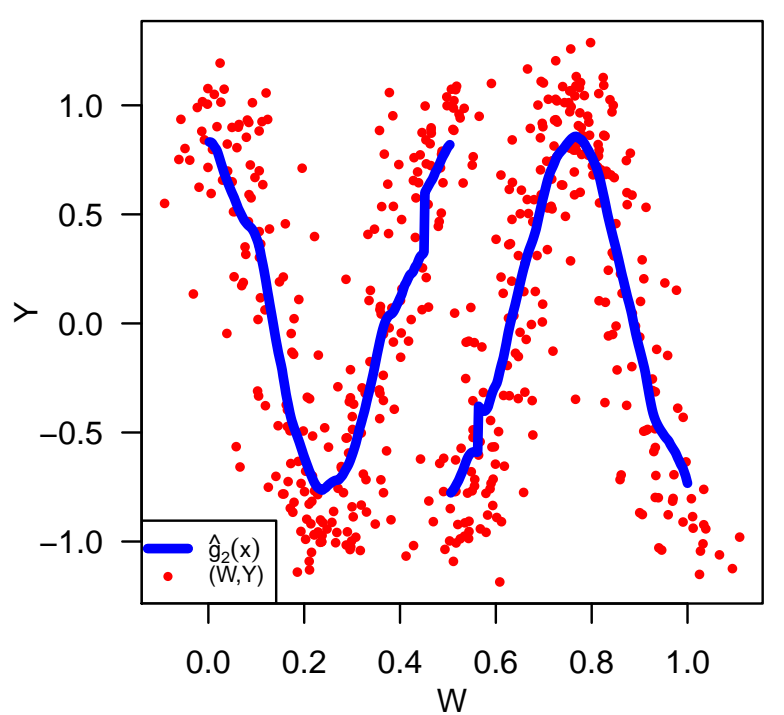

(d)

Figure 2: (a): $g_{1}$. (b): One realization with the regression function $g_{1}$ (dark dots) and $\widehat{g}_{1}$ (solid line). (c): $g_{2}$. (d): One realization with the regression function $g_{2}$ (dark dots) and $\widehat{g}_{2}$ (solid line). 
Table 2: Numerical comparison of the proposed method NEW with PLKE based on 200 replicated simulations. The numbers are in the unit of $10^{-3}$.

\begin{tabular}{lcccc} 
& \multicolumn{2}{c}{ NEW } & \multicolumn{2}{c}{ PLKE } \\
$f_{X}$ & AMSE & SDAMSE & AMSE & SDAMSE \\
Unif $[0,1]$ & 7.2116 & 0.1938 & 7.4668 & 0.1079 \\
$\operatorname{Beta}(2,2)$ & 14.2586 & 0.2717 & 20.4528 & 2.1102 \\
$\operatorname{Beta}(3,2)$ & 19.8202 & 0.5693 & 42.3686 & 4.3060 \\
$\operatorname{Beta}(2,3)$ & 23.2024 & 3.7319 & 25.5297 & 1.1948
\end{tabular}

Next, one realization of $\left\{\left(W_{i}, Y_{i}\right), i=1, \ldots, n\right\}$ when $f_{X}$ is Unif $[0,1]$ is shown in Fig. 3(a). The fitted curve by the proposed procedure and the one fitted by PLKE are shown together in Fig. 3(b). It can be seen that PLKE blurred the jump due to the impact of the measurement error whereas the proposed procedure preserves the jump well.

\section{Analysis of the Private Health Insurance Data}

In this section, we apply our proposed jump-preserving method to the private health insurance data for evaluating the impact of the medical levy surcharge policy on the take-up rate of private health insurance, as discussed in Section 1. The purposes of introducing private health insurance in Australia were to give consumers more choices and take some pressure off the public medical system. However, the private health insurance take-up rate by Australians was very low at the beginning when private health insurance was first introduced in 1984, and the take-up rate has been in declining until the end of 1990s (the take-up rate was only about 31 percent at that time) when a series of policies (including the medical levy surcharge) were introduced. Impact of some of these policy measures (e.g., Lifetime Health Cover) has been studied in a few studies, including Butler (2002), Frech et al. (2003), Palangkaraya and Yong (2005), and Palangkaraya et al. (2009). But the role of the medical levy surcharge has not been identified separately yet. The data used here are from a confidentialised "1\% Sample Unit Record File of Individual Income Tax Returns" for the 


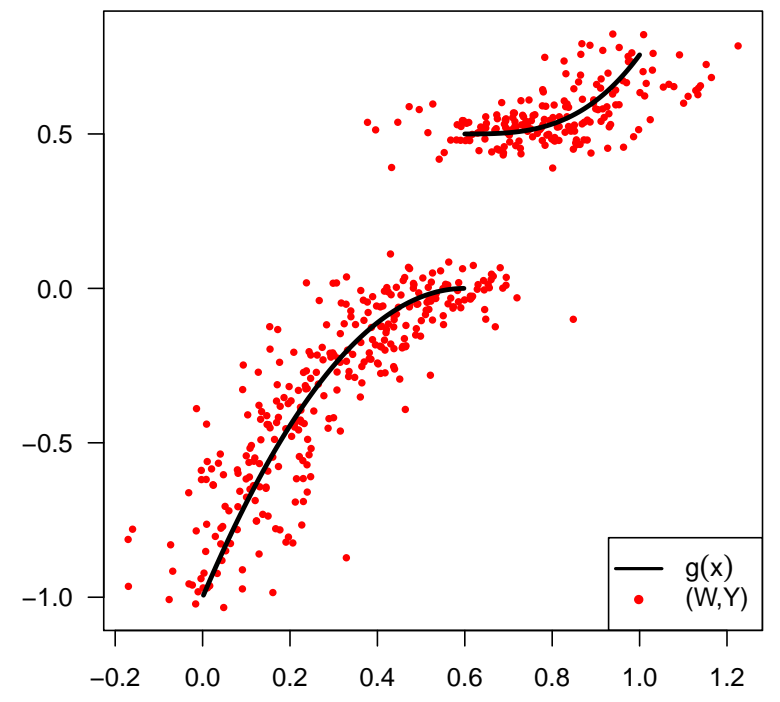

(a)

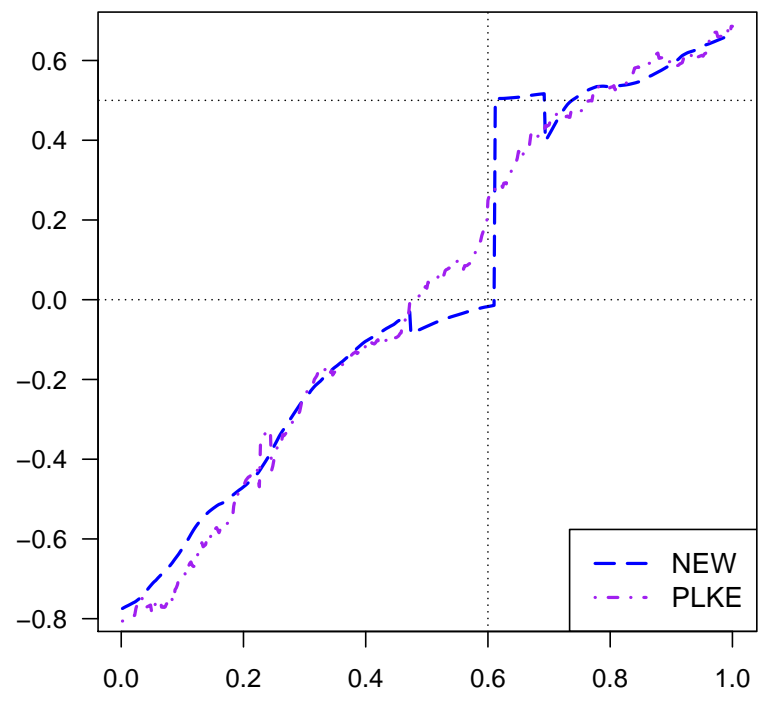

(b)

Figure 3: (a): $g(x)$ (solid line) and one realization of $\left\{\left(W_{i}, Y_{i}\right), i=1, \ldots, n\right\}$ (dark dots). (b): Estimated curves by NEW (long-dashed line) and by PLKE (dot-dashed line), respectively. The dotted lines mark the jump location (i.e., $x_{s}=0.6$ ) and the one-sided limits $\lim _{x \rightarrow x_{s} \pm} g(x)$. 
2003-04 financial year, that was developed by Australian Tax Office for research purposes. As a method of confidentialisation, Australian Tax Office 'perturbed' the income variables and did not reveal much information about the perturbation. The final sample for analysis contains 9,685 records of individual tax returns. See Kang et al. (2015) for more details about the data. By a preliminary analysis, we found that about $26 \%$ singles bought private health insurance in 2003-04, and the private health insurance take-up rates for those whose annual taxable incomes were below $\$ 50,000$ and those whose annual taxable incomes were above that level were quite different. The private health insurance take-up rate for the former group was about 21\%, and it was about $57 \%$ for the latter group. Because Australian Tax Office perturbed the income data by multiplying each original income observation by a random number, we used the income variable in log scale in our analysis, so that the additive measurement error assumption in (2) is valid here. Also, the response variable is 0 when an individual did not purchase private health insurance in 2003-04 and 1 otherwise. We transformed binned observations to meet the model assumption in (1) that the response variable is continuous numerical. Specifically, the bin size is chosen to be 40 . For each bin, the average of annual taxable income on $\log$ scale in that bin is used as the value for the new explanatory variable. And the log odds of the private health insurance take-up rate (i.e., $\log (p(x) /(1-p(x))$, where $p(x)$ denotes the private health insurance take-up rate when the bin average of the logarithm of the annual taxable income is $x$ ) is used as the transformed response variable. The log odds in the $i$ th bin is computed by

$$
\log \left(\frac{N_{i}+c}{m-N_{i}+c}\right),
$$

where $m=40$ is the bin size, $N_{i}$ denotes the number of people in the $i$ th bin who purchased private health insurance during 2003-04, and $c$ is some positive constant to avoid the numerical instabilities in computing the log odds. (12) is known as the empirical logistic transformation when $c=0.5$ and it yields some good statistical properties (see Cox 1970 for a detailed discussion). This choice for $c$ is also adopted here. The transformed private health insurance data is shown in Fig. 4 (dark dots). From the figure, it can be seen that there is an abrupt change in the log odds of private health insurance take-up rate within $[10.75,11.25]$ 
(i.e., the annual income is within $[\$ 36315, \$ 59874])$. The impact of the measurement error is also visible.

We then apply our proposed estimation procedure (3)-(9) to the transformed private health insurance data. The bandwidth and the threshold are chosen to be 0.135 and 0.13 , respectively. The results are shown in Fig. 4 (dashed line). From the plot, the abrupt change in the $\log$ odds is estimated to be at $10.99(\approx \$ 59,278)$. The $95 \%$ confidence interval given in Kang et al. (2015) is $(10.88,11.02)$. This finding confirms our intuition that people usually act later than they are hit by the medical levy surcharge. From Fig. 4, it can also be seen that the jump size is around 0.4945 in $\log$ odds $(\approx 12.3 \%$ in the private health insurance take-up rate). Kang et al. (2015) estimates the jump size to be 19\%. The difference could be resulted from the binning procedure we applied to transform the data. This number shows that the impact of the medical levy surcharge tax policy is substantial. For individuals with only one income source, the policy can be considered locally exogenous because the observations to the left and right of (but close to) the jump position are more or less homogeneous except the policy treatment. It implies that, among the individuals whose annual taxable income is around $\$ 59,278$, the medical levy surcharge policy brings about an extra $12.3 \%$ of them onto the private health system. This also implies a negative price elasticity of private health insurance demand since the jump in the take-up rate can be seen as a response to a price discount in the premium.

\section{Concluding Remarks}

We have proposed a jump-preserving curve estimation method when the explanatory variable has measurement error involved. A major feature of the proposed method is that it preserves jumps well without requiring much prior knowledge on the measurement error distribution, making it applicable in practice. The challenge caused by measurement error with an unknown distribution is handled by locally clustering of observations by maximizing a separation measure. Also, the proposed method is a direct approach without explicitly detecting the jump points beforehand. Thus, it is easy to use. 


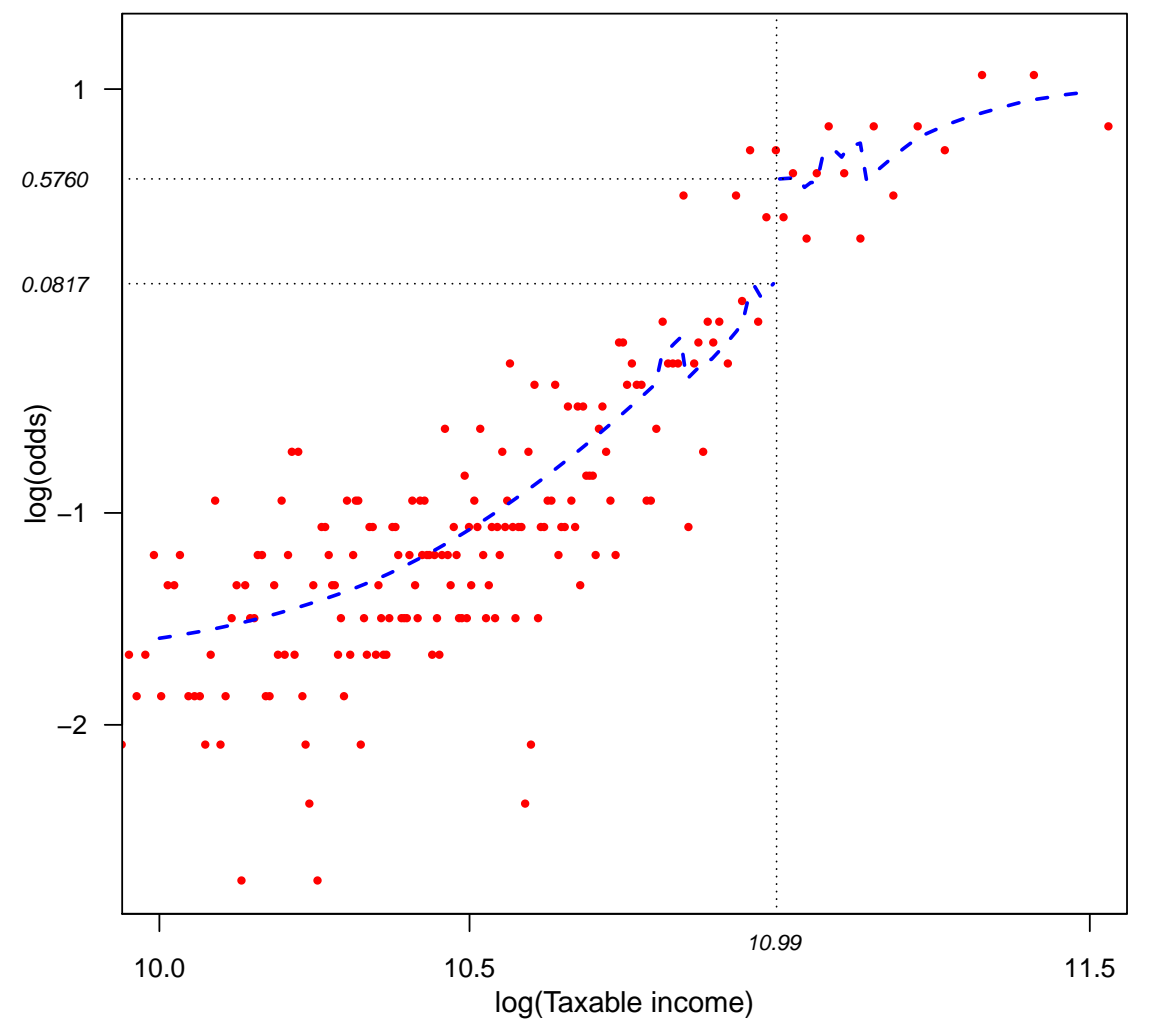

Figure 4: The estimated $\log (p(\cdot) /(1-p(\cdot)))$ (dashed line) and the observations of the private health insurance data after transformation (solid dots). 
There is much room for further improvement of the current method. First, estimated curve by the proposed method exhibits kinky behavior near jump points due to the hard thresholding. Some post-processing modifications may help improve the fitted curve. Second, the regression function at continuity points is estimated by the conventional local linear kernel smoothing procedure and the measurement error is ignored in such cases. It might be possible to estimate the distribution of the measurement error to some extent by making use of the jump structure of the regression function and then refine our estimate of the regression function in continuity regions. Fourth, the proposed bootstrap parameter selection procedure is evaluated by numerical studies only. It requires future research to derive the theoretical justification of its asymptotic behavior.

\section{Supplementary Material}

In this supplemental file, we mainly give the outline of the proof of Theorem 1 . Without loss of generality, assume that $g$ has a single jump at $x_{s} \in(0,1)$. For any given $x \in\left[h_{n}, 1-h_{n}\right]$, by some routine algebra, the solution to $(a, b)$ in $(3)$, denoted as $\left(\widehat{a}_{n}(x), \widehat{b}_{n}(x)\right)$, has the following expression:

$$
\begin{aligned}
\widehat{a}_{n}(x) & =\frac{\sum_{i=1}^{n} Y_{i} K\left(\frac{W_{i}-x}{h_{n}}\right)\left[Z_{2}(x)-Z_{1}(x)\left(W_{i}-x\right)\right]}{Z_{0}(x) Z_{2}(x)-Z_{1}(x)^{2}}, \\
\widehat{b}_{n}(x) & =\frac{\sum_{i=1}^{n} Y_{i} K\left(\frac{W_{i}-x}{h_{n}}\right)\left[Z_{0}(x)\left(W_{i}-x\right)-Z_{1}(x)\right]}{Z_{0}(x) Z_{2}(x)-Z_{1}(x)^{2}},
\end{aligned}
$$

where $Z_{k}(x)=\sum_{i=1}^{n}\left(W_{i}-x\right)^{k} K\left(\left(W_{i}-x\right) / h_{n}\right)$ for $k=0,1$, and 2 . We will denote $v_{k}=$ $\int_{-1}^{1} w^{k} K(w) d w$ throughout this supplemental material.

Lemma 2. Under the conditions of Theorem 1, for some $\gamma>0$ and some $\beta \in(0,1 / 4)$, we have the following:

$$
\sup _{x \in[0,1]}\left|Q_{l, k}(x)-E\left[Q_{l, k}(x)\right]\right|=o\left(\frac{(\log n)^{1+\gamma}}{\left(n h_{n}\right)^{\beta}}\right) \quad \text { a.s. },
$$

where

$$
Q_{l, k}(x)=\frac{1}{n h_{n}} \sum_{i=1}^{n} Y_{i}^{l}\left(\frac{W_{i}-x}{h_{n}}\right)^{k} K\left(\frac{W_{i}-x}{h_{n}}\right) \quad(l=0,1 ; k=0,1,2) .
$$


Proof. Define

$$
g_{n}(i)=\frac{1}{n h_{n}} Y_{i}^{l}\left(\frac{W_{i}-x}{h_{n}}\right)^{k} K\left(\frac{W_{i}-x}{h_{n}}\right) \quad(i=1, \ldots, n) .
$$

We first prove (S.13) holds for $l=0$. Notice the following fact:

$$
\begin{aligned}
& \mathrm{E}\left[\frac{Z_{k}(x)}{\left.n h_{n}^{k+1}\right]}-v_{k} f_{X}(x)\right. \\
= & v_{k+1} f_{X}^{\prime}(x) h_{n}+\frac{v_{k+2}}{2} f_{X}^{\prime \prime}(x) h_{n}^{2}+\frac{v_{k}}{2} f_{X}^{\prime \prime}(x) \sigma_{n}^{2}+O\left(h_{n}^{3}+\sigma_{n}^{3}\right),
\end{aligned}
$$

which follows from some straightforward calculation that

$$
\begin{aligned}
& \mathrm{E}\left[\frac{Z_{k}(x)}{n h_{n}^{k+1}}\right]-v_{k} f_{X}(x) \\
= & \frac{1}{h_{n}} \mathrm{E}\left[\left(\frac{W_{i}-x}{h_{n}}\right)^{k} K\left(\frac{W_{i}-x}{h_{n}}\right)\right]-v_{k} f_{X}(x) \\
= & \int_{-1}^{1} \int_{-\infty}^{\infty} w^{k} K(w) f_{X}\left(x+w h_{n}-\sigma_{n} u\right) f_{U}(u) d u d w-\int_{-1}^{1} w^{k} K(w) d w \cdot f_{X}(x) \\
= & \int_{-1}^{1} \int_{-\infty}^{\infty} w^{k} K(w)\left[f_{X}\left(x+w h_{n}-\sigma_{n} u\right)-f_{X}(x)\right] f_{U}(u) d u d w \\
= & \int_{-1}^{1} \int_{-\infty}^{\infty} w^{k} K(w)\left[f_{X}^{\prime}(x)\left(w h_{n}-\sigma_{n} u\right)+\frac{f_{X}^{\prime \prime}(x)}{2}\left(w h_{n}-\sigma_{n} u\right)^{2}+O\left(\left(w h_{n}-\sigma_{n} u\right)^{3}\right)\right] \\
& f_{U}(u) d u d w \\
= & v_{k+1} f_{X}^{\prime}(x) h_{n}+\frac{v_{k+2}}{2} f_{X}^{\prime \prime}(x) h_{n}^{2}+\frac{v_{k}}{2} f_{X}^{\prime \prime}(x) \sigma_{n}^{2}+O\left(h_{n}^{3}+\sigma_{n}^{3}\right),
\end{aligned}
$$

where $\mathrm{E}(U)=0$ and $\operatorname{var}(U)=1$ is used in the last equation. Thus (S.14) holds. Let $\xi_{n}=\left(n h_{n}\right)^{1 / 2}$ and for any $\epsilon>0$ consider the following:

$$
\begin{aligned}
& \operatorname{pr}\left(\frac{\xi_{n}}{(\log n)^{1+\gamma}}\left[\sum_{i=1}^{n} g_{n}(i)-\mathrm{E}\left(g_{n}(i)\right)\right]>\epsilon\right) \\
\leq & \exp \left[-\epsilon(\log n)^{1+\gamma}\right] \mathrm{E}\left\{\exp \left[\xi_{n} \sum_{i=1}^{n} g_{n}(i)-\mathrm{E}\left(g_{n}(i)\right)\right]\right\} \\
= & n^{-\epsilon(\log n)^{\gamma}} \prod_{i=1}^{n} \mathrm{E}\left\{\exp \left\{\xi_{n}\left[g_{n}(i)-\mathrm{E}\left(g_{n}(i)\right)\right]\right\}\right\}
\end{aligned}
$$


Notice that

$$
\begin{aligned}
& \left|\xi_{n}\left[g_{n}(i)-\mathrm{E}\left(g_{n}(i)\right)\right]\right| \\
= & \left|\left(n h_{n}\right)^{1 / 2}\left\{\frac{1}{n h_{n}}\left(\frac{W_{i}-x}{h_{n}}\right)^{k} K\left(\frac{W_{i}-x}{h_{n}}\right)-\mathrm{E}\left[\frac{1}{n h_{n}}\left(\frac{W_{i}-x}{h_{n}}\right)^{k} K\left(\frac{W_{i}-x}{h_{n}}\right)\right]\right\}\right| \\
\leq & \frac{1}{\left(n h_{n}\right)^{1 / 2}} C_{1},
\end{aligned}
$$

where we have used the fact that $K(\cdot)$ is bounded with support $[-1,1]$ and $C_{1}$ is some positive constant unrelated to $x$. By (S.16) and the fact that $e^{z} \leq 1+z+z^{2}$ for $|z| \leq 1 / 2$, we have

$$
\begin{aligned}
(\mathrm{S} .15) & \leq n^{-\epsilon(\log n)^{\gamma}} \prod_{i=1}^{n} \mathrm{E}\left\{1+\xi_{n}\left[g_{n}(i)-\mathrm{E} g_{n}(i)\right]+\xi_{n}^{2}\left[g_{n}(i)-\mathrm{E} g_{n}(i)\right]^{2}\right\} \\
& =n^{-\epsilon(\log n)^{\gamma}} \prod_{i=1}^{n}\left\{1+\operatorname{var}\left[\xi_{n} g_{n}(i)\right]\right\} \\
& \leq n^{-\epsilon(\log n)^{\gamma}} \exp \left\{\sum_{i=1}^{n} \operatorname{var}\left[\xi_{n} g_{n}(i)\right]\right\} \\
& =n^{-\epsilon(\log n)^{\gamma}} \exp \left\{n \xi_{n}^{2} \operatorname{var}\left[g_{n}(1)\right]\right\} \\
& \leq n^{-\epsilon(\log n)^{\gamma}} \exp \left\{n h_{n} n \frac{1}{n^{2} h_{n}^{2}} \mathrm{E}\left[\left(\frac{W_{i}-x}{h_{n}}\right)^{2 k} K\left(\frac{W_{i}-x}{h_{n}}\right)^{2 k}\right]\right\} \\
& =n^{-\epsilon(\log n)^{\gamma}} \exp \left[\int_{-1}^{1} w^{2 k} K(w) d w \cdot f_{X}(x)+O\left(h_{n}+\sigma_{n}^{2}\right)\right] \\
& \leq n^{-\epsilon(\log n)^{\gamma}} C_{2},
\end{aligned}
$$

where $C_{2}$ is some positive constant unrelated to $x$. By the same arguments, it can also be shown that

$$
\operatorname{pr}\left\{\frac{\xi_{n}}{(\log n)^{1+\gamma}}\left[\sum_{i=1}^{n} \mathrm{E}\left(g_{n}(i)\right)-g_{n}(i)\right]>\epsilon\right\} \leq n^{-\epsilon(\log n)^{\gamma}} C_{2} .
$$

Therefore, it follows from Borel-Cantelli Lemma that

$$
\frac{\left(n h_{n}\right)^{1 / 2}}{(\log n)^{1+\gamma}}\left|\frac{Z_{k}(x)}{n h_{n}^{k+1}}-\mathrm{E}\left[\frac{Z_{k}(x)}{n h_{n}^{k+1}}\right]\right| \rightarrow 0, \quad \text { a.s. }(n \rightarrow \infty) .
$$


And thus (S.13) holds for $l=0$ by the condition that $n h_{n} \rightarrow \infty$. Next, we prove (S.13) is also true for $l=1$. Let

$$
\tilde{Y}_{i}=Y_{i} \mathbb{1}_{\left\{\left|Y_{i}\right|<i^{\frac{1}{2}+\alpha}\right\}}, \quad \widetilde{g}_{n}(i)=\frac{1}{n h_{n}} \widetilde{Y}_{i}\left(\frac{W_{i}-x}{h_{n}}\right)^{k} K\left(\frac{W_{i}-x}{h_{n}}\right),
$$

where $\alpha>0$ is a very small number to be determined later, $k=0,1,2$, and $i=1,2, \ldots$ Let $\zeta_{n}=\left(n h_{n}\right)^{\beta}$. For any given $\epsilon>0$, We have

$$
\begin{aligned}
& \operatorname{pr}\left\{\frac{\zeta_{n}}{(\log n)^{1+\gamma}}\left[\sum_{i=1}^{n} \widetilde{g}_{n}(i)-\mathrm{E}\left[\widetilde{g}_{n}(i)\right]>\epsilon\right\}\right. \\
\leq & \exp \left[-\epsilon(\log n)^{1+\gamma}\right] \mathrm{E}\left\{\exp \left\{\zeta_{n}\left[\sum_{i=1}^{n} \widetilde{g}_{n}(i)-\mathrm{E}\left[\widetilde{g}_{n}(i)\right]\right]\right\}\right\} \\
= & n^{-\epsilon(\log n)^{\gamma}} \prod_{i=1}^{n} \mathrm{E}\left\{\exp \left[\zeta_{n}\left[\widetilde{g}_{n}(i)-\mathrm{E}\left[\widetilde{g}_{n}(i)\right]\right]\right]\right\}
\end{aligned}
$$

$$
\begin{aligned}
& \left|\zeta_{n}\left(\widetilde{g}_{n}(i)-\mathrm{E} \widetilde{g}_{n}(i)\right)\right| \\
= & \left(n h_{n}\right)^{\beta}\left|\frac{1}{n h_{n}} \widetilde{Y}_{i}\left(\frac{W_{i}-x}{h_{n}}\right)^{k} K\left(\frac{W_{i}-x}{h_{n}}\right)-\frac{1}{n h_{n}} \mathrm{E}\left[\widetilde{Y}_{i}\left(\frac{W_{i}-x}{h_{n}}\right)^{k} K\left(\frac{W_{i}-x}{h_{n}}\right)\right]\right| \\
\leq & C_{3}\left(n h_{n}\right)^{\beta} \frac{1}{n h_{n}} n^{\frac{1}{2}+\alpha} \\
= & C_{3} \frac{1}{n^{\frac{1}{2}-\alpha-\beta} h_{n}^{1-\beta}},
\end{aligned}
$$

where $C_{3}$ is some positive constant. If $\alpha$ is chosen such that $0.5-3 \alpha=2 \beta$ (need to have $\beta<1 / 4)$, then $(\mathrm{S} .18)=o(1)$ by the condition that $n^{1 / 3} h_{n} \rightarrow \infty$. By $e^{z} \leq 1+z+z^{2}$ for $|z| \leq 1 / 2$, we have

$$
\begin{aligned}
(\mathrm{S} .17) & \leq n^{-\epsilon(\log n)^{\gamma}} \prod_{i=1}^{n}\left\{1+\zeta_{n}^{2} \operatorname{var}\left[\widetilde{g}_{n}(i)\right]\right\} \\
& \leq n^{-\epsilon(\log n)^{\gamma}} \exp \left\{\sum_{i=1}^{n} \zeta_{n}^{2} \operatorname{var}\left[\widetilde{g}_{n}(i)\right]\right\} \\
& \leq n^{-\epsilon(\log n)^{\gamma}} \exp \left\{\sum_{i=1}^{n} \frac{\zeta_{n}^{2}}{n^{2} h_{n}^{2}} \mathrm{E}\left[\widetilde{Y}_{i}^{2}\left(\frac{W_{i}-x}{h_{n}}\right)^{2 k} K\left(\frac{W_{i}-x}{h_{n}}\right)^{2}\right]\right\} \\
& \leq n^{-\epsilon(\log n)^{\gamma}} \exp \left\{\sum_{i=1}^{n} \frac{\zeta_{n}^{2}}{n^{2} h_{n}^{2}} \mathrm{E}\left[Y_{i}^{2}\left(\frac{W_{i}-x}{h_{n}}\right)^{2 k} K\left(\frac{W_{i}-x}{h_{n}}\right)^{2}\right]\right\}
\end{aligned}
$$




$$
\begin{aligned}
& =n^{-\epsilon(\log n)^{\gamma}} \exp \left\{\mathrm{E}\left[\frac{n^{2 \beta} h_{n}^{2 \beta}}{n^{2} h_{n}^{2}} \sum_{i=1}^{n} \mathrm{E}\left(Y_{i}^{2} \mid X_{i}\right) \mathrm{E}\left(\left(\frac{W_{i}-x}{h_{n}}\right)^{2 k} K^{2}\left(\frac{W_{i}-x}{h_{n}}\right) \mid X_{i}\right)\right]\right\} \\
& \leq n^{-\epsilon(\log n)^{\gamma}} \exp \left\{\frac{C_{4}}{n^{1-2 \beta} h_{n}^{2-2 \beta}} \mathrm{E}\left[\left(\frac{W_{1}-x}{h_{n}}\right)^{2 k} K^{2}\left(\frac{W_{1}-x}{h_{n}}\right)\right]\right\} \\
& =n^{-\epsilon(\log n)^{\gamma}} \exp \left[\frac{C_{4}}{n^{1-2 \beta} h_{n}^{1-2 \beta}} \int_{-\infty}^{\infty} \int_{-\infty}^{\infty} w^{2 k} K^{2}(w) f_{X}\left(x+w h_{n}-\sigma_{n} u\right) f_{U}(u) d w d u\right] \\
& \leq n^{-\epsilon(\log n)^{\gamma}} \exp \left[\frac{C_{5}}{\left(n h_{n}\right)^{1-2 \beta}}\right],
\end{aligned}
$$

where the boundedness of $g(\cdot)$ is used in the second last inequality, the boundedness of $f_{X}(\cdot)$ and its derivatives is used in the last inequality, and $C_{4}$ and $C_{5}$ are positive constants unrelated to $x$. Similar calculation is performed in the last equation as did in (S.14). By the same arguments, we also have

$$
\operatorname{pr}\left\{\frac{\zeta_{n}}{(\log n)^{1+\gamma}}\left(\sum_{i=1}^{n} \mathrm{E}\left[\widetilde{g}_{n}(i)\right]-\widetilde{g}_{n}(i)\right)>\epsilon\right\} \leq n^{-\epsilon(\log n)^{\gamma}} \exp \left[\frac{C_{5}}{\left(n h_{n}\right)^{1-2 \beta}}\right] .
$$

By Borel-Cantelli Lemma,

$$
\frac{\zeta_{n}}{(\log n)^{1+\gamma}}\left|\sum_{i=1}^{n} \widetilde{g}_{n}(i)-\mathrm{E}\left[\widetilde{g}_{n}(i)\right]\right| \rightarrow 0 \text { a.s., as } n \rightarrow \infty .
$$

Next, we look at

$$
\begin{aligned}
& \frac{\zeta_{n}}{(\log n)^{1+\gamma}}\left[\sum_{i=1}^{n} g_{n}(i)-\widetilde{g}_{n}(i)\right] \\
&= \frac{1}{\left(n h_{n}\right)^{1-\beta}(\log n)^{1+\gamma}} \sum_{i=1}^{n}\left(Y_{i}-\widetilde{Y}_{i}\right)\left(\frac{W_{i}-x}{h_{n}}\right)^{k} K\left(\frac{W_{i}-x}{h_{n}}\right) . \\
& \mathrm{E}\left[\sum_{i=1}^{\infty} \mathbb{1}_{\left\{Y_{i} \neq \widetilde{Y}_{i}\right\}}\right]=\sum_{i=1}^{\infty} \operatorname{pr}\left(\left|Y_{i}\right|>i^{\frac{1}{2}+\alpha}\right) \leq \sum_{i=1}^{\infty} \frac{1}{i^{1+2 \alpha}} \mathrm{E}\left(Y_{i}^{2}\right)<\infty,
\end{aligned}
$$

where $\mathbb{1}_{\{\cdot\}}$ denotes the indicator function. Thus, there exists an $\Omega_{0}$ such that $\operatorname{pr}\left(\Omega_{0}\right)=1$ and that for each $\omega \in \Omega_{0}$, there exists an $N_{\omega}>0$,

$$
Y_{n}(w)=\widetilde{Y}_{n}(w) \quad\left(n \geq N_{\omega}\right)
$$


By (S.21), we have

$$
\frac{\zeta_{n}}{(\log n)^{1+\gamma}}\left[\sum_{i=1}^{n} g_{n}(i)-\widetilde{g}_{n}(i)\right] \rightarrow 0, \quad \text { a.s. }(n \rightarrow \infty) .
$$

Also, we have

$$
\begin{aligned}
& \left|\frac{\zeta_{n}}{(\log n)^{1+\gamma}} \sum_{i=1}^{n} \mathrm{E}\left[g_{n}(i)-\widetilde{g}_{n}(i)\right]\right| \\
= & \frac{1}{\left(n h_{n}\right)^{1-\beta}(\log n)^{1+\gamma}}\left|\sum_{i=1}^{n} \mathrm{E}\left[Y_{i} \mathbb{1}_{\left\{\left|Y_{i}\right|>i^{\frac{1}{2}+\alpha}\right\}}\left(\frac{W_{i}-x}{h_{n}}\right)^{k} K\left(\frac{W_{i}-x}{h_{n}}\right)\right]\right| \\
\leq & \frac{1}{\left(n h_{n}\right)^{1-\beta}(\log n)^{1+\gamma}} \sum_{i=1}^{n} \frac{1}{i^{\frac{1}{2}+\alpha}} \mathrm{E}\left[Y_{i}^{2}\left(\frac{W_{i}-x}{h_{n}}\right)^{k} K\left(\frac{W_{i}-x}{h_{n}}\right)\right] \\
= & \frac{1}{\left(n h_{n}\right)^{1-\beta}(\log n)^{1+\gamma}} \sum_{i=1}^{n} \frac{1}{i^{\frac{1}{2}+\alpha}} \mathrm{E}\left[\mathrm{E}\left(Y_{i}^{2} \mid X_{i}\right) \mathrm{E}\left(\frac{W_{i}-x}{h_{n}}\right)^{k} K\left(\frac{W_{i}-x}{h_{n}} \mid X_{i}\right)\right] \\
\leq & \frac{C_{6}}{\left(n h_{n}\right)^{1-\beta}(\log n)^{1+\gamma}} \sum_{i=1}^{n} \frac{1}{i^{\frac{1}{2}+\alpha}} \mathrm{E}\left[\left(\frac{W_{i}-x}{h_{n}}\right)^{k} K\left(\frac{W_{i}-x}{h_{n}}\right)\right] \\
= & \frac{C_{6}}{\left(n h_{n}\right)^{1-\beta}(\log n)^{1+\gamma}} \sum_{i=1}^{n} \frac{h_{n}}{i^{\frac{1}{2}+\alpha}} \int_{-\infty}^{\infty} \int_{-\infty}^{\infty} w^{k} K(w) f_{X}\left(x+w h_{n}-\sigma_{n}\right) f_{U}(u) d w d u \\
\leq & \frac{C_{7} h_{n}^{\beta}}{n^{1-\beta}(\log n)^{1+\gamma}} n^{\frac{1}{2}-\alpha} \\
= & C_{7} \frac{h_{n}^{\beta}}{n^{\frac{1}{2}-(\alpha+\beta)}(\log n)^{1+\gamma}},
\end{aligned}
$$

where the boundedness of $g(\cdot)$ is used in the second last inequality, the boundedness of $f_{X}(\cdot)$ and its derivatives is used the last inequality, and $C_{6}$ and $C_{7}$ are some positive constants unrelated to $x$. Since $\alpha+\beta<1 / 2$, we have (S.23)=o(1) as well. By (S.19), (S.22), and (S.23), we have

$$
\begin{aligned}
\frac{\left(n h_{n}\right)^{\beta}}{(\log n)^{1+\gamma}} & \left|\frac{1}{n h_{n}} \sum_{i=1}^{n} Y_{i}\left(\frac{W_{i}-x}{h_{n}}\right)^{k} K\left(\frac{W_{i}-x}{h_{n}}\right)-\mathrm{E}\left[Y_{i}\left(\frac{W_{i}-x}{h_{n}}\right)^{k} K\left(\frac{W_{i}-x}{h_{n}}\right)\right]\right| \\
& \rightarrow 0 \text { a.s., }
\end{aligned}
$$

as $n \rightarrow \infty$. Therefore, (S.13) holds for $l=1$. And it completes the proof of Lemma 2 . 
Lemma 3. Suppose that $\left|x-x_{s}\right| \geq \delta$ for some $\delta>0$. Then,

$$
\begin{aligned}
\widehat{a}_{n}(x) & =g(x)+O\left(h_{n}^{2}+\sigma_{n}^{2}+\frac{(\log n)^{1+\gamma}}{\left(n h_{n}\right)^{\beta}}\right) \text { a.s. } \\
\widehat{b}_{n}(x) & =g^{\prime}(x)+O\left(h_{n}+\frac{\sigma_{n}^{2}}{h_{n}}+\frac{(\log n)^{1+\gamma}}{\left(n h_{n}\right)^{\beta} h_{n}}\right) \text { a.s. } \\
\operatorname{WRMS}_{n}(x) & =\tau^{2}+O\left(h_{n}^{2}+\sigma_{n}^{2}+\frac{(\log n)^{1+\gamma}}{\left(n h_{n}\right)^{\beta}}\right) \text { a.s. }
\end{aligned}
$$

Proof. First, we write

$$
\widehat{a}_{n}(x)=\frac{\frac{Z_{2}(x)}{n h_{n}^{3}} \frac{1}{n h_{n}} \sum_{i=1}^{n} Y_{i} K\left(\frac{W_{i}-x}{h_{n}}\right)-\frac{Z_{1}(x)}{n h_{n}^{2}} \frac{1}{n h_{n}} \sum_{i=1}^{n} Y_{i} \frac{W_{i}-x}{h_{n}} K\left(\frac{W_{i}-x}{h_{n}}\right)}{\frac{Z_{0}(x)}{n h_{n}} \frac{Z_{2}(x)}{n h_{n}^{3}}-\frac{Z_{1}(x)}{n h_{n}^{2}} \frac{Z_{1}(x)}{n h_{n}^{2}}} .
$$

Denote $m(\cdot)=g(\cdot) f_{X}(\cdot)$. We have that

$$
\begin{aligned}
\mathrm{E}\left[\frac{1}{n h_{n}} \sum_{i=1}^{n} Y_{i} K\left(\frac{W_{i}-x}{h_{n}}\right)\right] & =\frac{1}{h_{n}} \int_{-\infty}^{\infty} \int_{-\infty}^{\infty} g(v) K\left(\frac{v+\sigma_{n} u-x}{h_{n}}\right) f_{X}(v) f_{U}(u) d u d v \\
& =\int_{-\infty}^{\infty} \int_{-\infty}^{\infty} m\left(x+w h_{n}-\sigma_{n} u\right) K(w) f_{U}(u) d u d w
\end{aligned}
$$

There exists $x_{L}<x_{U}$ such that $x \in\left[x_{L}, x_{U}\right],\left|x_{L}-x_{s}\right| \geq \delta / 2$, and $\left|x_{U}-x_{s}\right| \geq \delta / 2$. Then we can continue the calculation as follows.

$$
\begin{aligned}
(\mathrm{S} .25)= & \int_{-1}^{1} K(w) d w \cdot\left[\int_{\frac{x_{L}-x-w h_{n}}{\sigma_{n}}}^{\frac{x_{U}-x-w h_{n}}{\sigma_{n}}} m\left(x+w h_{n}-\sigma_{n} u\right) f_{U}(u) d u\right. \\
& \left.+\int_{-\infty}^{\frac{x_{L}-x-w h_{n}}{\sigma_{n}}} m\left(x+w h_{n}-\sigma_{n} u\right) f_{U}(u) d u+\int_{\frac{x_{U}-x-w h_{n}}{\sigma_{n}}}^{\infty} m\left(x+w h_{n}-\sigma_{n} u\right) f_{U}(u) d u\right] \\
= & \int_{-1}^{1} K(w) d u \int_{\frac{x_{L}-x-w h_{n}}{\sigma_{n}}}^{\frac{x_{U}-x-w h_{n}}{\sigma_{n}}}\left[m(x)+m^{\prime}(x)\left(w h_{n}-\sigma_{n} u\right)+\frac{1}{2} m^{\prime \prime}(x)\right. \\
& \left.\cdot\left(w h_{n}-\sigma_{n} u\right)^{2}+\frac{1}{6} m^{\prime \prime \prime}\left(\xi_{n}\right)\left(w h_{n}-\sigma_{n} u\right)^{3}\right] f_{U}(u) d u d w+O\left(\sigma_{n}^{2}\right) \\
= & v_{0} m(x)+\frac{1}{2} m^{\prime \prime}(x) v_{2} h_{n}^{2}+\frac{1}{2} m^{\prime \prime}(x) v_{0} \sigma_{n}^{2}+O\left(h_{n}^{3}+\sigma_{n}^{3}\right)+O\left(\sigma_{n}^{2}\right)
\end{aligned}
$$


where $\xi_{n}$ is some real number, $\int_{|u|>x} f_{U}(u) d u=O\left(1 / x^{2}\right)^{1}$ is used in the second last equation, and the boundedness of $m(\cdot)$ and its derivatives, $\mathrm{E}(U)=0$, and $\operatorname{var}(U)=1$ are used in the last equation. By Lemma 2 and $v_{3}=0$, we have

$$
\begin{aligned}
\frac{Z_{2}(x)}{n h_{n}^{3}}= & v_{2} f_{X}(x)+v_{3} f_{X}^{\prime}(x) h_{n}^{2}+\frac{1}{2} v_{4} f_{X}^{\prime \prime}(x) h_{n}^{2}+\frac{1}{2} v_{2} f_{X}^{\prime \prime}(x) \sigma_{n}^{2}+O\left(h_{n}^{3}+\sigma_{n}^{3}\right)+ \\
& o\left(\frac{(\log n)^{1+\gamma}}{\left(n h_{n}\right)^{\beta}}\right) \\
= & v_{2} f_{X}(x)+\frac{1}{2} v_{4} f_{X}^{\prime \prime}(x) h_{n}^{2}+\frac{1}{2} v_{2} f_{X}^{\prime \prime}(x) \sigma_{n}^{2}+O\left(h_{n}^{3}+\sigma_{n}^{3}\right)+o\left(\frac{(\log n)^{1+\gamma}}{\left(n h_{n}\right)^{\beta}}\right) \text { a.s. }
\end{aligned}
$$

Thus,

$$
\begin{aligned}
& \frac{Z_{2}(x)}{n h_{n}^{3}} \frac{1}{n h_{n}} \sum_{i=1}^{n} Y_{i} K\left(\frac{W_{i}-x}{h_{n}}\right) \\
= & v_{2} v_{0} f_{X}(x) m(x)+\frac{1}{2} h_{n}^{2}\left[v_{0} v_{4} f_{X}^{\prime \prime}(x) m(x)+v_{2}^{2} m^{\prime \prime}(x) f_{X}(x)\right]+\frac{1}{2} \sigma_{n}^{2} v_{0} v_{2} . \\
& {\left[f_{X}^{\prime \prime}(x) m(x)+f_{X}(x) m^{\prime \prime}(x)\right]+O\left(h_{n}^{2}+\sigma_{n}^{2}\right)+o\left(\frac{(\log n)^{1+\gamma}}{\left(n h_{n}\right)^{\beta}}\right) \quad \text { a.s. } }
\end{aligned}
$$

Next, we calculate the following

$$
\begin{aligned}
& \mathrm{E}\left[\frac{1}{n h_{n}} \sum_{i=1}^{n} Y_{i} \frac{W_{i}-x}{h_{n}} K\left(\frac{W_{i}-x}{h_{n}}\right)\right] \\
= & \frac{1}{n h_{n}} \sum_{i=1}^{n} \mathrm{E}\left\{\mathrm{E}\left[Y_{i} \mid X_{i}\right] \mathrm{E}\left[\frac{W_{i}-x}{h_{n}} K\left(\frac{W_{i}-x}{h_{n}}\right) \mid X_{i}\right]\right\} \\
= & \frac{1}{n h_{n}} \sum_{i=1}^{n} \mathrm{E}\left\{m\left(X_{i}\right) \mathrm{E}\left[\frac{W_{i}-x}{h_{n}} K\left(\frac{W_{i}-x}{h_{n}}\right) \mid X_{i}\right]\right\} \\
= & \frac{1}{n h_{n}} \sum_{i=1}^{n} \mathrm{E}\left\{\mathrm{E}\left[m\left(X_{i}\right) \frac{W_{i}-x}{h_{n}} K\left(\frac{W_{i}-x}{h_{n}}\right) \mid X_{i}\right]\right\} \\
= & \frac{1}{n h_{n}} \sum_{i=1}^{n} \mathrm{E}\left[m\left(X_{i}\right) \frac{W_{i}-x}{h_{n}} K\left(\frac{W_{i}-x}{h_{n}}\right)\right] \\
= & \frac{1}{h_{n}} \mathrm{E}\left[m\left(X_{1}\right) \frac{W_{1}-x}{h_{n}} K\left(\frac{W_{1}-x}{h_{n}}\right)\right] \\
= & \int_{-\infty}^{\infty} \int_{-\infty}^{\infty} w K(w) m\left(x+w h_{n}-\sigma_{n} u\right) f_{U}(u) d u d w
\end{aligned}
$$

${ }^{1}$ It follows from that $\int_{|u|>x} f_{U}(u) d u \leq \int_{|u|>x} u^{2} / x^{2} f_{U}(u) d u \leq 1 / x^{2} \operatorname{var}(U)=1 / x^{2}$. 


$$
\begin{aligned}
= & \int_{-1}^{1} w K(w)\left\{\int _ { \frac { x _ { L } - x - w h _ { n } } { \sigma _ { n } } } ^ { \frac { x _ { U } - x - w h _ { n } } { \sigma _ { n } } } \left[m(x)+m^{\prime}(x)\left(w h_{n}-\sigma_{n} u\right)+\frac{1}{2} m^{\prime \prime}(x)\left(w h_{n}-\sigma_{n} u\right)^{2}\right.\right. \\
& \left.\left.+O\left(h_{n}^{3}+\sigma_{n}^{3}\right)\right] f_{U}(u) d u\right\} d w+O\left(\sigma_{n}^{2}\right) \\
= & v_{1} m(x)+v_{2} m^{\prime}(x) h_{n}+\frac{v_{3}}{2} m^{\prime \prime}(x) h_{n}^{2}+\frac{v_{1}}{2} m^{\prime \prime}(x) \sigma_{n}^{2}+O\left(h_{n}^{2}+\sigma_{n}^{2}\right) \\
= & v_{2} m^{\prime}(x) h_{n}+O\left(h_{n}^{2}+\sigma_{n}^{2}\right) .
\end{aligned}
$$

By Lemma 2, we have

$$
\frac{1}{n h_{n}} \sum_{i=1}^{n} Y_{i} \frac{W_{i}-x}{h_{n}} K\left(\frac{W_{i}-x}{h_{n}}\right)=v_{2} m^{\prime}(x) h_{n}+O\left(h_{n}^{2}+\sigma_{n}^{2}+\frac{(\log n)^{1+\gamma}}{\left(n h_{n}\right)^{\beta}}\right) \text { a.s. }
$$

Also, by (S.14), we have

$$
\begin{aligned}
\frac{Z_{1}(x)}{n h_{n}^{2}} & =v_{1} f_{X}(x)+v_{2} f_{X}^{\prime}(x) h_{n}+\frac{v_{3}}{2} f_{X}^{\prime \prime}(x) h_{n}^{2}+\frac{v_{1}}{2} f_{X}^{\prime \prime}(x) \sigma_{n}^{2}+o\left(h_{n}^{2}+\sigma_{n}^{2}+\frac{(\log n)^{1+\gamma}}{\left(n h_{n}\right)^{\beta}}\right) \\
& =v_{2} f_{X}^{\prime}(x) h_{n}+o\left(h_{n}^{2}+\sigma_{n}^{2}+\frac{(\log n)^{1+\gamma}}{\left(n h_{n}\right)^{\beta}}\right) \text { a.s. }
\end{aligned}
$$

Then, by (S.29) - (S.30),

$$
\frac{Z_{1}(x)}{n h_{n}^{2}} \frac{1}{n h_{n}} \sum_{i=1}^{n} Y_{i} \frac{W_{i}-x}{h_{n}} K\left(\frac{W_{i}-x}{h_{n}}\right)=v_{2}^{2} m^{\prime}(x) f_{X}^{\prime}(x) h_{n}^{2}+O\left(h_{n}^{2}+\sigma_{n}^{2}+\frac{(\log n)^{1+\gamma}}{\left(n h_{n}\right)^{\beta}}\right) \text { a.s. }
$$

By (S.28) and (S.31),

$$
\begin{aligned}
& \frac{Z_{2}(x)}{n h_{n}^{3}} \frac{1}{n h_{n}} \sum_{i=1}^{n} Y_{i} K\left(\frac{W_{i}-x}{h_{n}}\right)-\frac{Z_{1}(x)}{n h_{n}^{2}} \frac{1}{n h_{n}} \sum_{i=1}^{n} Y_{i} \frac{W_{i}-x}{h_{n}} K\left(\frac{W_{i}-x}{h_{n}}\right) \\
= & v_{0} v_{2} f_{X}(x) m(x)+\frac{h_{n}^{2}}{2}\left[v_{0} v_{4} f_{X}^{\prime \prime}(x) m(x)+v_{2}^{2} m^{\prime \prime}(x) f_{X}(x)-2 v_{2}^{2} m^{\prime}(x) f_{X}^{\prime}(x)\right] \\
& +\frac{v_{0} v_{2} \sigma_{n}^{2}}{2}\left[f_{X}^{\prime \prime}(x) m(x)+f_{X}(x) m^{\prime \prime}(x)\right]+o\left(h_{n}^{2}+\sigma_{n}^{2}+\frac{(\log n)^{1+\gamma}}{\left(n h_{n}\right)^{\beta}}\right) \text { a.s. }
\end{aligned}
$$

By Lemma 2, we have

$$
\begin{aligned}
& \frac{Z_{0}(x)}{n h_{n}} \frac{Z_{2}(x)}{n h_{n}^{3}}-\frac{Z_{1}(x)}{n h_{n}^{2}} \frac{Z_{1}(x)}{n h_{n}^{2}} \\
= & v_{0} v_{2} f_{X}(x)^{2}+\frac{h_{n}^{2}}{2}\left[v_{2}^{2} f_{X}^{\prime \prime}(x) f_{X}(x)+v_{0} v_{4} f_{X}^{\prime \prime}(x) f_{X}(x)-2 v_{2}^{2} f_{X}^{\prime}(x)^{2}\right] \\
& +v_{0} v_{2} \sigma_{n}^{2} f_{X}^{\prime \prime}(x) f(x)+o\left(h_{n}^{2}+\sigma_{n}^{2}+\frac{(\log n)^{1+\gamma}}{\left(n h_{n}\right)^{\beta}}\right) \text { a.s. }
\end{aligned}
$$


By (S.32) - (S.33), we have

$$
\begin{aligned}
\widehat{a}_{n}(x) & =\frac{\frac{Z_{2}(x)}{n h_{n}^{3}} \frac{1}{n h_{n}} \sum_{i=1}^{n} Y_{i} K\left(\frac{W_{i}-x}{h_{n}}\right)-\frac{Z_{1}(x)}{n h_{n}^{2}} \frac{1}{n h_{n}} \sum_{i=1}^{n} Y_{i} \frac{W_{i}-x}{h_{n}} K\left(\frac{W_{i}-x}{h_{n}}\right)}{\frac{Z_{0}(x)}{n h_{n}} \frac{Z_{2}(x)}{n h_{n}^{3}}-\frac{Z_{1}(x)}{n h_{n}^{2}} \frac{Z_{1}(x)}{n h_{n}^{2}}} \\
& =\frac{v_{0} v_{2} f_{X}(x) m(x)+O\left(h_{n}^{2}+\sigma_{n}^{2}+\frac{(\log n)^{1+\gamma}}{\left(n h_{n}\right)^{\beta}}\right)}{v_{0} v_{2} f_{X}(x)^{2}+O\left(h_{n}^{2}+\sigma_{n}^{2}+\frac{(\log n)^{1+\gamma}}{\left(n h_{n}\right)^{\beta}}\right)} \\
& =g(x)+O\left(h_{n}^{2}+\sigma_{n}^{2}+\frac{(\log n)^{1+\gamma}}{\left(n h_{n}\right)^{\beta}}\right) a . s .
\end{aligned}
$$

Similarly, we have

$$
\begin{aligned}
\widehat{b}_{n}(x) & =\frac{\frac{Z_{0}(x)}{n h_{n}} \frac{1}{n h_{n}} \sum_{i=1}^{n} Y_{i} \frac{W_{i}-x}{h_{n}} K\left(\frac{W_{i}-x}{h_{n}}\right)-\frac{Z_{1}(x)}{n h_{n}^{2}} \frac{1}{n h_{n}} \sum_{i=1}^{n} Y_{i} K\left(\frac{W_{i}-x}{h_{n}}\right)}{h_{n}\left[\frac{Z_{0}(x)}{n h_{n}} \frac{Z_{2}(x)}{n h_{n}^{3}}-\frac{Z_{1}(x)}{n h_{n}^{2}} \frac{Z_{1}(x)}{n h_{n}^{2}}\right]} \\
& =\frac{v_{0} v_{2} f_{X}(x) m^{\prime}(x) h_{n}-v_{0} v_{2} f_{X}^{\prime}(x) m(x) h_{n}+O\left(h_{n}^{2}+\sigma_{n}^{2}+\frac{(\log n)^{1+\gamma}}{\left(n h_{n}\right)^{\beta}}\right)}{h_{n}\left[v_{0} v_{2} f_{X}(x)^{2}+O\left(h_{n}^{2}+\sigma_{n}^{2}+\frac{(\log n)^{1+\gamma}}{\left(n h_{n}\right)^{\beta}}\right)\right]} \\
& =g^{\prime}(x)+O\left(h_{n}+\frac{\sigma_{n}^{2}}{h_{n}}+\frac{(\log n)^{1+\gamma}}{\left(n h_{n}\right)^{\beta} h_{n}}\right) \text { a.s. }
\end{aligned}
$$

It remains to calculate $\mathrm{WRMS}_{n}(x)$.

$$
\begin{aligned}
\operatorname{WRMS}_{n}(x)= & \frac{\sum_{i=1}^{n}\left[Y_{i}-\widehat{a}_{n}(x)-\widehat{b}_{n}(x)\left(W_{i}-x\right)\right]^{2} K\left(\frac{W_{i}-x}{h_{n}}\right)}{\sum_{i=1}^{n} K\left(\frac{W_{i}-x}{h_{n}}\right)} \\
= & \frac{\frac{1}{n h_{n}} \sum_{i=1}^{n} \varepsilon_{i}^{2} K\left(\frac{W_{i}-x}{h_{n}}\right)}{\frac{1}{n h_{n}} \sum_{i=1}^{n} K\left(\frac{W_{i}-x}{h_{n}}\right)} \\
& +2 \frac{\frac{1}{n h_{n}} \sum_{i=1}^{n} \varepsilon_{i}\left[g\left(X_{i}\right)-\widehat{a}_{n}(x)-\widehat{b}_{n}(x)\left(W_{i}-x\right)\right] K\left(\frac{W_{i}-x}{h_{n}}\right)}{\frac{1}{n h_{n}} \sum_{i=1}^{n} K\left(\frac{W_{i}-x}{h_{n}}\right)} \\
& +\frac{\frac{1}{n h_{n}} \sum_{i=1}^{n}\left[g\left(X_{i}\right)-\widehat{a}_{n}(x)-\widehat{b}_{n}(x)\left(W_{i}-x\right)\right]^{2} K\left(\frac{W_{i}-x}{h_{n}}\right)}{\frac{1}{n h_{n}} \sum_{i=1}^{n} K\left(\frac{W_{i}-x}{h_{n}}\right)}
\end{aligned}
$$

By the same arguments in Lemma 2, we can show that

$$
(\mathrm{S} .34)=\tau^{2}+O\left(h_{n}^{2}+\sigma_{n}^{2}+\frac{(\log n)^{1+\gamma}}{\left(n h_{n}\right)^{\beta}}\right) \text { a.s. }
$$


Also notice that, for each $i$, we have

$$
\begin{aligned}
& g\left(X_{i}\right)-\widehat{a}_{n}(x)-\widehat{b}_{n}(x)\left(W_{i}-x\right) \\
= & g(x)-\widehat{a}_{n}(x)+h_{n}\left[g^{\prime}(x)-\widehat{b}_{n}(x)\right] \frac{W_{i}-x}{h_{n}}-g^{\prime}(x) \sigma_{n} U_{i} \\
& +\frac{h_{n}^{2}}{2} g^{\prime \prime}(x)\left(\frac{W_{i}-x}{h_{n}}\right)^{2}+\frac{\sigma_{n}^{2}}{2} g^{\prime \prime}(x) U_{i}^{2}-h_{n} \sigma_{n} g^{\prime \prime}(x) \frac{W_{i}-x}{h_{n}} \\
& +o\left(\left(W_{i}-x\right)^{2}+\sigma_{n}^{2} U_{i}-2 \sigma_{n}\left(W_{i}-x\right)\right) .
\end{aligned}
$$

And

$$
\begin{aligned}
g(x)-\widehat{a}_{n}(x) & =O\left(h_{n}^{2}+\sigma_{n}^{2}+\frac{(\log n)^{1+\gamma}}{\left(n h_{n}\right)^{\beta}}\right) \text { a.s. } \\
h_{n}\left[g^{\prime}(x)-\widehat{b}_{n}(x)\right] & =O\left(h_{n}^{2}+\sigma_{n}^{2}+\frac{(\log n)^{1+\gamma}}{\left(n h_{n}\right)^{\beta}}\right) \text { a.s. }
\end{aligned}
$$

By the same arguments in Lemma 2, it can be shown that

$$
\begin{aligned}
\frac{1}{n h_{n}} \sum_{i=1}^{n} \varepsilon_{i} K\left(\frac{W_{i}-x}{h_{n}}\right) & =o\left(\frac{(\log n)^{1+\gamma}}{\left(n h_{n}\right)^{\beta}}\right) \text { a.s. } \\
\frac{1}{n h_{n}} \sum_{i=1}^{n} \varepsilon_{i} \frac{W_{i}-x}{h_{n}} K\left(\frac{W_{i}-x}{h_{n}}\right) & =o\left(\frac{(\log n)^{1+\gamma}}{\left(n h_{n}\right)^{\beta}}\right) \text { a.s. } \\
\frac{1}{n h_{n}} \sum_{i=1}^{n} \varepsilon_{i}\left(\frac{W_{i}-x}{h_{n}}\right)^{2} K\left(\frac{W_{i}-x}{h_{n}}\right) & =O\left(\frac{(\log n)^{1+\gamma}}{\left(n h_{n}\right)^{\beta}}\right) \text { a.s. } \\
\frac{1}{n h_{n}} \sum_{i=1}^{n} \varepsilon_{i} U_{i} K\left(\frac{W_{i}-x}{h_{n}}\right) & =o\left(\frac{(\log n)^{1+\gamma}}{\left(n h_{n}\right)^{\beta}}\right) \text { a.s. } \\
\frac{1}{n h_{n}} \sum_{i=1}^{n} \varepsilon_{i} U_{i}^{2} K\left(\frac{W_{i}-x}{h_{n}}\right) & =o\left(\frac{(\log n)^{1+\gamma}}{\left(n h_{n}\right)^{\beta}}\right) \text { a.s. }
\end{aligned}
$$

The above results can also be seen by applying Lemma 2 directly to the special case when $m(\cdot)=0$ (i.e., $Y_{i}=\varepsilon_{i}$ for each $\left.i=1,2, \cdots\right)$. Hence,

$$
\begin{aligned}
& (\mathrm{S} .35)=O\left(h_{n}^{2}+\sigma_{n}^{2}+\frac{(\log n)^{1+\gamma}}{\left(n h_{n}\right)^{\beta}}\right) \text { a.s. } \\
& (\mathrm{S} .36)=O\left(h_{n}^{4}+\sigma_{n}^{2}+\frac{(\log n)^{2+2 \gamma}}{\left(n h_{n}\right)^{2 \beta}}\right) \text { a.s. }
\end{aligned}
$$


Therefore,

$$
\mathrm{WRMS}_{n}(x)=\tau^{2}+O\left(h_{n}^{2}+\sigma_{n}^{2}+\frac{(\log n)^{1+\gamma}}{\left(n h_{n}\right)^{\beta}}\right) \text { a.s. }
$$

The proof of Lemma 3 has been completed.

Lemma 4. Suppose that $x_{s}=x+s \cdot h_{n}$ with $s \in(-1,1)$. Let $d_{s}=g_{+}\left(x_{s}\right)-g_{-}\left(x_{s}\right)$, where $g_{ \pm}\left(x_{s}\right)=\lim _{z \rightarrow x_{s}} g(z)$. We have the following results:

(i) If $\sigma_{n} / h_{n}=o(1)$, then

$$
\begin{aligned}
& \widehat{a}_{n}(x)=g_{-}\left(x_{s}\right)+d_{s} \int_{s}^{1} K(w) d w+O\left(\frac{\sigma_{n}}{h_{n}}+h_{n}+\frac{(\log n)^{1+\gamma}}{\left(n h_{n}\right)^{\beta}}\right) \text { a.s. } \\
& \widehat{b}_{n}(x)=d_{s} \frac{\int_{s}^{1} w K(w) d w}{h_{n} v_{2}}+O\left(\frac{\sigma_{n}}{h_{n}}+h_{n}+\frac{(\log n)^{1+\gamma}}{\left(n h_{n}\right)^{\beta}}\right) \text { a.s. } \\
& W R M S_{n}(x)=d_{s}^{2}\left[\int_{-1}^{s}\left(\int_{s}^{1} K(z) d z+\frac{w}{v_{2}} \int_{s}^{1} z K(z) d z\right)^{2} K(w) d w\right. \\
& \left.+\int_{s}^{1}\left(\int_{-1}^{s} K(z) d z-\frac{w}{v_{2}} \int_{s}^{1} z K(z) d z\right)^{2} K(w) d w\right] \\
& +\tau^{2}+O\left(\frac{\sigma_{n}}{h_{n}}+h_{n}+\frac{(\log n)^{1+\gamma}}{\left(n h_{n}\right)^{\beta}}\right) \text { a.s. }
\end{aligned}
$$

(ii) If $\sigma_{n} / h_{n} \rightarrow \infty$, then

$$
\begin{aligned}
& \widehat{a}_{n}(x)=\frac{1}{2}\left[g_{-}\left(x_{s}\right)+g_{+}\left(x_{s}\right)\right]+O\left(\sigma_{n}+\frac{h_{n}}{\sigma_{n}}+\frac{(\log n)^{1+\gamma}}{\left(n h_{n}\right)^{\beta}}\right), \text { a.s. } \\
& \widehat{b}_{n}(x)=\frac{1}{h_{n}} O\left(\frac{h_{n}}{\sigma_{n}}+\sigma_{n}+\frac{(\log n)^{1+\gamma}}{\left(n h_{n}\right)^{\beta}}\right), \text { a.s. } \\
& W_{R M S_{n}}(x)=\tau^{2}+\frac{d_{s}^{2}}{4}+O\left(\frac{h_{n}}{\sigma_{n}}+\sigma_{n}+\frac{(\log n)^{1+\gamma}}{\left(n h_{n}\right)^{\beta}}\right), \text { a.s. }
\end{aligned}
$$

(iii) If $\sigma_{n} / h_{n}=O(1)$, then

$$
\begin{aligned}
& \widehat{a}_{n}(x)=g_{-}\left(x_{s}\right)+d_{s} \int_{-\infty}^{\infty} f_{U}(u) \int_{s+u \frac{\sigma_{n}}{h_{n}}}^{1} K(w) d w+O\left(h_{n}+\sigma_{n}+\frac{(\log n)^{1+\gamma}}{\left(n h_{n}\right)^{\beta}}\right), \text { a.s. } \\
& \widehat{b}_{n}(x)=\frac{d_{s}}{h_{n}} \int_{-\infty}^{\infty} f_{U}(u) \int_{s+u \frac{\sigma_{n}}{h_{n}}}^{1} K(w) \frac{w}{v_{2}} d w+\frac{1}{h_{n}} O\left(h_{n}+\sigma_{n}+\frac{(\log n)^{1+\gamma}}{\left(n h_{n}\right)^{\beta}}\right), \text { a.s. }
\end{aligned}
$$




$$
\begin{aligned}
& W R M S_{n}(x)=d_{s}^{2}\left\{\int _ { - \infty } ^ { \infty } f _ { U } ( u ) \int _ { - 1 } ^ { s + u \frac { \sigma _ { n } } { h _ { n } } } \left[\int_{-\infty}^{\infty} f_{U}\left(u^{\prime}\right) \int_{s+u^{\prime} \frac{\sigma_{n}}{h_{n}}}^{1} K(z) d z d u^{\prime}\right.\right. \\
& \left.+\int_{-\infty}^{\infty} f_{U}\left(u^{\prime}\right) \int_{s+u^{\prime} \frac{\sigma_{n}}{h_{n}}}^{1} K(z) \frac{z}{v_{2}} d z d u^{\prime} \cdot w\right]^{2} K(w) d u d w \\
& +\int_{-\infty}^{\infty} f_{U}(u) \int_{s+u \frac{\sigma_{n}}{h_{n}}}^{1}\left[\int_{-\infty}^{\infty} f_{U}\left(u^{\prime}\right) \int_{-1}^{s+u^{\prime} \frac{\sigma_{n}}{h_{n}}} K(z) d z d u^{\prime}\right. \\
& \left.\left.-\int_{-\infty}^{\infty} f_{U}\left(u^{\prime}\right) \int_{s+u^{\prime} \frac{\sigma_{n}}{h_{n}}}^{1} K(z) \frac{z}{v_{2}} d z d u^{\prime} \cdot w\right]^{2} K(w) d u d w\right\} \\
& +\tau^{2}+O\left(h_{n}+\sigma_{n}+\frac{(\log n)^{1+\gamma}}{\left(n h_{n}\right)^{\beta}}\right) \text { a.s., a.s. }
\end{aligned}
$$

Proof. (i) We have that

$$
\begin{aligned}
& \widehat{a}_{n}(x)= \sum_{i=1}^{n} Y_{i} K\left(\frac{W_{i}-x}{h_{n}}\right) \frac{Z_{2}(x)-Z_{1}(x)\left(W_{i}-x\right)}{Z_{0}(x) Z_{2}(x)-Z_{1}(x)^{2}} \\
&= \sum_{i=1}^{n} g\left(X_{i}\right) K\left(\frac{W_{i}-x}{h_{n}}\right) \frac{Z_{2}(x)-Z_{1}(x)\left(W_{i}-x\right)}{Z_{0}(x) Z_{2}(x)-Z_{1}(x)^{2}} \\
&+\sum_{i=1}^{n} \varepsilon_{i} K\left(\frac{W_{i}-x}{h_{n}}\right) \frac{Z_{2}(x)-Z_{1}(x)\left(W_{i}-x\right)}{Z_{0}(x) Z_{2}(x)-Z_{1}(x)^{2}} \\
&= \sum_{X_{i}<x_{s}} g\left(X_{i}\right) K\left(\frac{W_{i}-x}{h_{n}}\right) \frac{Z_{2}(x)-Z_{1}(x)\left(W_{i}-x\right)}{Z_{0}(x) Z_{2}(x)-Z_{1}(x)^{2}} \\
&+\sum_{X_{i} \geq x_{s}} g\left(X_{i}\right) K\left(\frac{W_{i}-x}{h_{n}}\right) \frac{Z_{2}(x)-Z_{1}(x)\left(W_{i}-x\right)}{Z_{0}(x) Z_{2}(x)-Z_{1}(x)^{2}} \\
&+o\left(\frac{(\log n)^{1+\gamma}}{\left(n h_{n}\right)^{\beta}}\right) a_{. s .} \\
&=g_{-}\left(x_{s}\right) \int_{-\infty}^{\infty} \int_{-\infty}^{\infty} \mathbb{1}_{\left\{w h_{n}-\sigma_{n} u<s h_{n}\right\}} K(w) f_{X}\left(x+w h_{n}-\sigma_{n} u\right) f_{U}(u) d w d u+ \\
& \frac{1}{n h_{n}} \sum_{X_{i}<x_{s}} g_{-} x_{s} \frac{1}{n h_{n}} \sum_{X_{i}<x_{s}} \mathbb{1}_{\left\{X_{i}<x_{s}\right\}} K\left(\frac{W_{i}-x}{h_{n}}\right) \\
& x_{s}\left(\frac{W_{i}-x}{h_{n}}\right)
\end{aligned}
$$




$$
\begin{aligned}
& o\left(\frac{(\log n)^{1+\gamma}}{\left(n h_{n}\right)^{\beta}}\right) \\
= & g_{-}\left(x_{s}\right) \int_{-\infty}^{\infty} \int_{-\infty}^{s+\frac{\sigma_{n}}{h_{n}} u} K(w) f_{X}\left(x+w h_{n}-\sigma_{n} u\right) f_{U}(u) d w d u+o\left(\frac{(\log n)^{1+\gamma}}{\left(n h_{n}\right)^{\beta}}\right) \\
= & g_{-}\left(x_{s}\right) \int_{-\infty}^{\infty} f_{U}(u) \int_{-\infty}^{s} K(w) f_{X}\left(x+w h_{n}-\sigma_{n} u\right) d w d u \\
& +g_{-}\left(x_{s}\right) \int_{-\infty}^{\infty} f_{U}(u) \int_{s}^{s+\frac{\sigma_{n}}{h_{n}} u} K(w) f_{X}\left(x+w h_{n}-\sigma_{n} u\right) d w d u+o\left(\frac{(\log n)^{1+\gamma}}{\left(n h_{n}\right)^{\beta}}\right) \\
= & g_{-}\left(x_{s}\right) f_{X}(x) \int_{-1}^{s} K(w) d w+O\left(h_{n}+\sigma_{n}+\frac{\sigma_{n}}{h_{n}}+\frac{(\log n)^{1+\gamma}}{\left(n h_{n}\right)^{\beta}}\right) \text { a.s. }
\end{aligned}
$$

In the last equation, we have used the boundedness of $f_{X}(\cdot)$ and its derivatives and the boundedness of $K(\cdot)$. By Lemma 2, (S.37), and (S.14),

$$
\begin{aligned}
& \widehat{a}_{n}(x) \\
= & \sum_{X_{i}<x_{s}}\left\{\left[g_{-}\left(x_{s}\right)+g^{\prime}\left(\theta_{i}^{-}\right)\left(W_{i}-x-\sigma_{n} U_{i}\right)\right] K\left(\frac{W_{i}-x}{h_{n}}\right) \frac{Z_{2}(x)-Z_{1}(x)\left(W_{i}-x\right)}{Z_{0}(x) Z_{2}(x)-Z_{1}(x)^{2}}\right\} \\
& +\sum_{X_{i} \geq x_{s}}\left\{\left[g_{+}\left(x_{s}\right)+g^{\prime}\left(\theta_{i}^{+}\right)\left(W_{i}-x-\sigma_{n} U_{i}\right)\right] K\left(\frac{W_{i}-x}{h_{n}}\right) \frac{Z_{2}(x)-Z_{1}(x)\left(W_{i}-x\right)}{Z_{0}(x) Z_{2}(x)-Z_{1}(x)^{2}}\right\} \\
& +o\left(\frac{(\log n)^{1+\gamma}}{\left(n h_{n}\right)^{\beta}}\right) \\
= & g_{-}\left(x_{s}\right) \int_{-1}^{s} K(w) \frac{v_{2}-v_{1} w}{v_{0} v_{2}-v_{1}^{2}} d w+g_{+}\left(x_{s}\right) \int_{s}^{1} K(w) \frac{v_{2}-v_{1} w}{v_{0} v_{2}-v_{1}^{2}} d w \\
& +O\left(h_{n}+\sigma_{n}+\frac{\sigma_{n}}{h_{n}}+\frac{(\log n)^{1+\gamma}}{\left(n h_{n}\right)^{\beta}}\right) \\
= & g_{-}\left(x_{s}\right)+d_{s} \int_{s}^{1} K(w) \frac{v_{2}-v_{1} w}{v_{0} v_{2}-v_{1}^{2}} d w+O\left(h_{n}+\sigma_{n}+\frac{\sigma_{n}}{h_{n}}+\frac{(\log n)^{1+\gamma}}{\left(n h_{n}\right)^{\beta}}\right) \\
= & g_{-}\left(x_{s}\right)+d_{s} \int_{s}^{1} K(w) d w+O\left(h_{n}+\frac{\sigma_{n}}{h_{n}}+\frac{(\log n)^{1+\gamma}}{\left(n h_{n}\right)^{\beta}}\right) a . s .,
\end{aligned}
$$

where $\theta_{i}^{-}$and $\theta_{i}^{+}$are some numbers between $X_{i}$ and $x_{s}$ for each $i=1,2, \cdots, n$. Next, we calculate

$$
\widehat{b}_{n}(x)
$$




$$
\begin{aligned}
& =\sum_{i=1}^{n} Y_{i} K\left(\frac{W_{i}-x}{h_{n}}\right) \frac{Z_{0}(x)\left(W_{i}-x\right)-Z_{1}(x)}{Z_{0}(x) Z_{2}(x)-Z_{1}^{2}(x)} \\
& =\sum_{i=1}^{n} g\left(X_{i}\right) K\left(\frac{W_{i}-x}{h_{n}}\right) \frac{Z_{0}(x)\left(W_{i}-x\right)-Z_{1}(x)}{Z_{0}(x) Z_{2}(x)-Z_{1}^{2}(x)}+ \\
& \sum_{i=1}^{n} \varepsilon_{i} K\left(\frac{W_{i}-x}{h_{n}}\right) \frac{Z_{0}(x)\left(W_{i}-x\right)-Z_{1}(x)}{Z_{0}(x) Z_{2}(x)-Z_{1}^{2}(x)} \\
& =\sum_{X_{i}<x_{s}} g\left(X_{i}\right) K\left(\frac{W_{i}-x}{h_{n}}\right) \frac{Z_{0}(x)\left(W_{i}-x\right)-Z_{1}(x)}{Z_{0}(x) Z_{2}(x)-Z_{1}^{2}(x)} \\
& +\sum_{X_{i} \geq x_{s}} g\left(X_{i}\right) K\left(\frac{W_{i}-x}{h_{n}}\right) \frac{Z_{0}(x)\left(W_{i}-x\right)-Z_{1}(x)}{Z_{0}(x) Z_{2}(x)-Z_{1}^{2}(x)}+O\left(\frac{(\log n)^{1+\gamma}}{\left(n h_{n}\right)^{\beta} h_{n}}\right) \\
& =\sum_{X_{i}<x_{s}}\left\{\left[g_{-}\left(x_{s}\right)+g^{\prime}\left(\theta_{i}^{-}\right)\left(W_{i}-x-\sigma_{n} U_{i}\right)\right] \cdot K\left(\frac{W_{i}-x}{h_{n}}\right) \frac{Z_{2}(x)-Z_{1}(x)\left(W_{i}-x\right)}{Z_{0}(x) Z_{2}(x)-Z_{1}(x)^{2}}\right\} \\
& +\sum_{X_{i} \geq x_{s}}\left\{\left[g_{+}\left(x_{s}\right)+g^{\prime}\left(\theta_{i}^{+}\right)\left(W_{i}-x-\sigma_{n} U_{i}\right)\right] \cdot K\left(\frac{W_{i}-x}{h_{n}}\right) \frac{Z_{2}(x)-Z_{1}(x)\left(W_{i}-x\right)}{Z_{0}(x) Z_{2}(x)-Z_{1}(x)^{2}}\right\} \\
& +o\left(\frac{(\log n)^{1+\gamma}}{\left(n h_{n}\right)^{\beta} h_{n}}\right) \\
& =\sum_{X_{i}<x_{s}} g_{-}\left(x_{s}\right) K\left(\frac{W_{i}-x}{h_{n}}\right) \frac{Z_{0}(x)\left(W_{i}-x\right)-Z_{1}(x)}{Z_{0}(x) Z_{2}(x)-Z_{1}^{2}(x)} \\
& +\sum_{X_{i} \geq x_{s}} g_{+}\left(x_{s}\right) K\left(\frac{W_{i}-x}{h_{n}}\right) \frac{Z_{0}(x)\left(W_{i}-x\right)-Z_{1}(x)}{Z_{0}(x) Z_{2}(x)-Z_{1}^{2}(x)}+\frac{1}{h_{n}} O\left(h_{n}+\sigma_{n}+\frac{(\log n)^{1+\gamma}}{\left(n h_{n}\right)^{\beta}}\right) \\
& =\frac{g_{-}\left(x_{s}\right)}{h_{n} f_{X}(x)}\left[v_{0} \int_{-\infty}^{\infty} \int_{-\infty}^{\infty} \mathbb{1}_{\left\{w-u \frac{\sigma_{n}}{h_{n}}<s\right\}} K(w) w f_{X}\left(x+w h_{n}-\sigma_{n} u\right) f_{U}(u) d w d u-\right. \\
& \left.v_{1} \int_{-\infty}^{\infty} \int_{-\infty}^{\infty} \mathbb{1}_{\left\{w-u \frac{\sigma_{n}}{h_{n}}<s\right\}} K(w) f_{X}\left(x+w h_{n}-\sigma_{n} u\right) f_{U}(u) d w d u\right] \frac{1}{\left(v_{0} v_{2}-v_{1}^{2}\right)} \\
& +\frac{g_{+}\left(x_{s}\right)}{h_{n} f_{X}(x)}\left[v_{0} \int_{-\infty}^{\infty} \int_{-\infty}^{\infty} \mathbb{1}_{\left\{w-u \frac{\sigma_{n}}{h_{n}} \geq s\right\}} K(w) w f_{X}\left(x+w h_{n}-\sigma_{n} u\right) f_{U}(u) d w d u-\right. \\
& \left.v_{1} \int_{-\infty}^{\infty} \int_{-\infty}^{\infty} \mathbb{1}_{\left\{w-u \frac{\sigma_{n}}{h_{n}} \geq s\right\}} K(w) f_{X}\left(x+w h_{n}-\sigma_{n} u\right) f_{U}(u) d w d u\right] \frac{1}{\left(v_{0} v_{2}-v_{1}^{2}\right)} \\
& +\frac{1}{h_{n}} O\left(h_{n}+\sigma_{n}+\frac{(\log n)^{1+\gamma}}{\left(n h_{n}\right)^{\beta}}\right) \\
& =\frac{g_{-}\left(x_{s}\right)}{h_{n}\left(v_{0} v_{2}-v_{1}^{2}\right)}\left[v_{0} \int_{-\infty}^{\infty} f_{U}(u) \int_{-1}^{s+u \frac{\sigma_{n}}{h_{n}}} w K(w) d w d u-v_{1} \int_{-\infty}^{\infty} f_{U}(u) \int_{-1}^{s+u \frac{\sigma_{n}}{h_{n}}} K(w) d w d u\right]
\end{aligned}
$$




$$
\begin{aligned}
& +\frac{g_{+}\left(x_{s}\right)}{h_{n}\left(v_{0} v_{2}-v_{1}^{2}\right)}\left[v_{0} \int_{-\infty}^{\infty} f_{U}(u) \int_{s+u \frac{\sigma_{n}}{h_{n}}}^{1} w K(w) d w d u-v_{1} \int_{-\infty}^{\infty} f_{U}(u) \int_{s+u \frac{\sigma_{n}}{h_{n}}}^{1} K(w) d w d u\right] \\
& +\frac{1}{h_{n}} O\left(h_{n}+\sigma_{n}+\frac{(\log n)^{1+\gamma}}{\left(n h_{n}\right)^{\beta}}\right) \\
= & g_{-}\left(x_{s}\right) \int_{-1}^{s} \frac{v_{0} w K(w)-v_{1} K(w)}{h_{n}\left(v_{0} v_{2}-v_{1}^{2}\right)} d w+g_{+}\left(x_{s}\right) \int_{s}^{1} \frac{v_{0} w K(w)-v_{1} K(w)}{h_{n}\left(v_{0} v_{2}-v_{1}^{2}\right)} d w \\
& +\frac{1}{h_{n}} O\left(\frac{\sigma_{n}}{h_{n}}+h_{n}+\sigma_{n}+\frac{(\log n)^{1+\gamma}}{\left(n h_{n}\right)^{\beta}}\right) \\
= & \frac{d_{s}}{h_{n}} \int_{s}^{1} \frac{v_{0} w K(w)-v_{1} K(w)}{h_{n}\left(v_{0} v_{2}-v_{1}^{2}\right)} d w+\frac{1}{h_{n}} O\left(\frac{\sigma_{n}}{h_{n}}+h_{n}+\sigma_{n}+\frac{(\log n)^{1+\gamma}}{\left(n h_{n}\right)^{\beta}}\right) \\
= & \frac{d_{s}}{h_{n}} \int_{s}^{1} \frac{w K(w)}{h_{n} v_{2}} d w+\frac{1}{h_{n}} O\left(\frac{\sigma_{n}}{h_{n}}+h_{n}+\frac{(\log n)^{1+\gamma}}{\left(n h_{n}\right)^{\beta}}\right) \text { a.s. }
\end{aligned}
$$

It remains to calculate $\mathrm{WRMS}_{n}(x)$ to complete the proof of (i).

$$
\mathrm{WRMS}_{n}(x)=(\mathrm{S} .34)+(\mathrm{S} .35)+(\mathrm{S} .36) .
$$

And we have

$$
(\mathrm{S} .34)=\tau^{2}+O\left(h_{n}^{2}+\sigma_{n}^{2}+\frac{(\log n)^{1+\gamma}}{\left(n h_{n}\right)^{\beta}}\right) \text { a.s. }
$$

Next, by similar arguments in Lemma 2

$$
\begin{aligned}
(\mathrm{S} .35)= & \frac{\frac{2}{n h_{n}} \sum_{i=1}^{n} \varepsilon_{i} g\left(X_{i}\right) K\left(\frac{W_{i}-x}{h_{n}}\right)}{\frac{1}{n h_{n}} \sum_{i=1}^{n} K\left(\frac{W_{i}-x}{h_{n}}\right)}-\frac{\frac{2}{n h_{n}} \sum_{i=1}^{n} \varepsilon_{i} \widehat{a}_{n}(x) K\left(\frac{W_{i}-x}{h_{n}}\right)}{\frac{1}{n h_{n}} \sum_{i=1}^{n} K\left(\frac{W_{i}-x}{h_{n}}\right)} \\
& -\frac{\frac{2}{n h_{n}} h_{n} \widehat{b}_{n}(x) \sum_{i=1}^{n} \varepsilon_{i} \frac{W_{i}-x}{h_{n}} K\left(\frac{W_{i}-x}{h_{n}}\right)}{\frac{1}{n h_{n}} \sum_{i=1}^{n} K\left(\frac{W_{i}-x}{h_{n}}\right)} \\
= & O\left(\frac{(\log n)^{1+\gamma}}{\left(n h_{n}\right)^{\beta}}\right) \text { a.s. }
\end{aligned}
$$

Next, we calculate

$$
\begin{aligned}
& \frac{1}{n h_{n}} \sum_{i=1}^{n}\left[g\left(X_{i}\right)-\widehat{a}_{n}(x)-\widehat{b}_{n}(x)\left(W_{i}-x\right)\right]^{2} K\left(\frac{W_{i}-x}{h_{n}}\right) \\
= & \frac{1}{n h_{n}}\left\{\sum_{X_{i}<x_{s}}+\sum_{X_{i} \geq x_{s}}\right\}\left[g\left(X_{i}\right)-\widehat{a}_{n}(x)-h_{n} \widehat{b}_{n}(x) \frac{W_{i}-x}{h_{n}}\right]^{2} K\left(\frac{W_{i}-x}{h_{n}}\right)
\end{aligned}
$$




$$
\begin{aligned}
& =\frac{1}{n h_{n}} \sum_{X_{i}<x_{s}}\left[g_{-}\left(x_{s}\right)+g^{\prime}\left(\theta_{i}^{-}\right)\left(W_{i}-x\right)-\widehat{a}_{n}(x)-h_{n} \widehat{b}_{n}(x) \frac{W_{i}-x}{h_{n}}-g^{\prime}\left(\theta_{i}^{-}\right) \sigma_{n} U_{i}\right]^{2} \\
& K\left(\frac{W_{i}-x}{h_{n}}\right) \\
& +\frac{1}{n h_{n}} \sum_{X_{i} \geq x_{s}}\left[g_{+}\left(x_{s}\right)+g^{\prime}\left(\theta_{i}^{+}\right)\left(W_{i}-x\right)-\widehat{a}_{n}(x)-h_{n} \widehat{b}_{n}(x) \frac{W_{i}-x}{h_{n}}-g^{\prime}\left(\theta_{i}^{+}\right) \sigma_{n} U_{i}\right]^{2} \\
& K\left(\frac{W_{i}-x}{h_{n}}\right) \\
& =\frac{1}{n h_{n}} \sum_{X_{i}<x_{s}}\left[g_{-}\left(x_{s}\right)-\widehat{a}_{n}(x)-h_{n} \widehat{b}_{n}(x) \frac{W_{i}-x}{h_{n}}\right]^{2} K\left(\frac{W_{i}-x}{h_{n}}\right) \\
& +\frac{1}{n h_{n}} \sum_{X_{i} \geq x_{s}}\left[g_{+}\left(x_{s}\right)-\widehat{a}_{n}(x)-h_{n} \widehat{b}_{n}(x) \frac{W_{i}-x}{h_{n}}\right]^{2} K\left(\frac{W_{i}-x}{h_{n}}\right)+O\left(h_{n}+\sigma_{n}\right) \\
& =\frac{1}{n h_{n}} \sum_{X_{i}<x_{s}}\left[d_{s} \int_{s}^{1} K(w) \frac{v_{2}-v_{1} w}{v_{0} v_{2}-v_{1}^{2}} d w+d_{s} \int_{s}^{1} \frac{v_{0} w-v_{1}}{v_{0} v_{2}-v_{1}^{2}} K(w) d w \frac{W_{i}-x}{h_{n}}\right]^{2} \\
& \cdot K\left(\frac{W_{i}-x}{h_{n}}\right)+\frac{1}{n h_{n}} \sum_{X_{i} \geq x_{s}}\left[d_{s} \int_{-1}^{s} K(w) \frac{v_{2}-v_{1} w}{v_{0} v_{2}-v_{1}^{2}} d w-d_{s} \int_{s}^{1} \frac{v_{0} w-v_{1}}{v_{0} v_{2}-v_{1}^{2}} K(w)\right. \\
& \left.d w \frac{W_{i}-x}{h_{n}}\right]^{2} K\left(\frac{W_{i}-x}{h_{n}}\right)+O\left(\frac{\sigma_{n}}{h_{n}}+h_{n}+\sigma_{n}+\frac{(\log n)^{1+\gamma}}{\left(n h_{n}\right)^{\beta}}\right) \\
& =d_{s}^{2} \int_{-\infty}^{\infty} \int_{-\infty}^{\infty} \mathbb{1}_{\left\{w<s+\frac{\sigma_{n}}{h_{n}}\right\}}\left[\int_{s}^{1} K(z) \frac{v_{2}-v_{1} z}{v_{0} v_{2}-v_{1}^{2}} d z+\int_{s}^{1} K(z) \frac{v_{0} z-v_{1}}{v_{0} v_{2}-v_{1}^{2}} d z \cdot w\right]^{2} . \\
& K(w) f_{X}\left(x+w h_{n}-\sigma_{n} u\right) f_{U}(u) d u d w+d_{s}^{2} \int_{-\infty}^{\infty} \int_{\infty}^{\infty} \mathbb{1}_{\left\{w \geq s+u \frac{\sigma_{n}}{h_{n}}\right\}}\left[\int_{-1}^{s} K(z) \frac{v_{2}-v_{1} z}{v_{0} v_{2}-v_{1}^{2}} d z\right. \\
& \left.-\int_{s}^{1} K(z) \frac{v_{0} z-v_{1}}{v_{0} v_{2}-v_{1}^{2}} d z \cdot w\right]^{2} K(w) f_{X}\left(x+w h_{n}-\sigma_{n} u\right) f_{U}(u) d w d u \\
& +O\left(\frac{\sigma_{n}}{h_{n}}+h_{n}+\sigma_{n}+\frac{(\log n)^{1+\gamma}}{\left(n h_{n}\right)^{\beta}}\right) \\
& =f_{X}(x) d_{s}^{2}\left[\int_{-1}^{s}\left(\int_{s}^{1} K(z) \frac{v_{2}-v_{1} z}{v_{0} v_{2}-v_{1}^{2}} d z+\int_{s}^{1} K(z) \frac{v_{0} z-v_{1}}{v_{0} v_{2}-v_{1}^{2}} d z \cdot w\right)^{2} K(w) d w\right. \\
& \left.+\int_{s}^{1}\left(\int_{-1}^{s} K(z) \frac{v_{2}-v_{1} z}{v_{0} v_{2}-v_{1}^{2}} d z-\int_{s}^{1} K(z) \frac{v_{0} z-v_{1}}{v_{0} v_{2}-v_{1}^{2}} d z \cdot w\right)^{2} K(w) d w\right]
\end{aligned}
$$




$$
+O\left(\frac{\sigma_{n}}{h_{n}}+h_{n}+\sigma_{n}+\frac{(\log n)^{1+\gamma}}{\left(n h_{n}\right)^{\beta}}\right) \text { a.s. }
$$

Therefore,

$$
\begin{aligned}
\operatorname{WRMS}_{n}(x)=d_{s}^{2} & {\left[\int_{-1}^{s}\left(\int_{s}^{1} K(z) \frac{v_{2}-v_{1} z}{v_{0} v_{2}-v_{1}^{2}} d z+\int_{s}^{1} K(z) \frac{v_{0} z-v_{1}}{v_{0} v_{2}-v_{1}^{2}} d z \cdot w\right)^{2} K(w) d w\right.} \\
& \left.+\int_{s}^{1}\left(\int_{-1}^{s} K(z) \frac{v_{2}-v_{1} z}{v_{0} v_{2}-v_{1}^{2}} d z-\int_{s}^{1} K(z) \frac{v_{0} z-v_{1}}{v_{0} v_{2}-v_{1}^{2}} d z \cdot w\right)^{2} K(w) d w\right] \\
& \left.+\tau^{2}+O\left(\frac{\sigma_{n}}{h_{n}}+h_{n}+\sigma_{n}+\frac{(\log n)^{1+\gamma}}{\left(n h_{n}\right)^{\beta}}\right)^{2}\right)^{2} K(w) d w \\
= & d_{s}^{2}\left[\int_{-1}^{s}\left(\int_{s}^{1} K(z) d z+\int_{s}^{1} K(z) \frac{z}{v_{2}} d z \cdot w\right)^{2} K(w) d w\right] \\
& +\int_{s}^{1}\left(\int_{-1}^{s} K(z) d z-\int_{s}^{1} K(z) \frac{z}{v_{2}} d z \cdot w\right)^{2} K(w) \\
& +\tau^{2}+O\left(\frac{\sigma_{n}}{h_{n}}+h_{n}+\frac{(\log n)^{1+\gamma}}{\left(n h_{n}\right)^{\beta}}\right) a . s .
\end{aligned}
$$

(ii) First we calculate $\widehat{a}_{n}(x)$ as follows.

$$
\begin{aligned}
& \widehat{a}_{n}(x) \\
= & \sum_{i=1}^{n} Y_{i} K\left(\frac{W_{i}-x}{h_{n}}\right) \frac{Z_{2}(x)-Z_{1}(x)\left(W_{i}-x\right)}{Z_{0}(x) Z_{2}(x)-Z_{1}^{2}(x)} \\
= & \sum_{i=1}^{n} g\left(X_{i}\right) K\left(\frac{W_{i}-x}{h_{n}}\right) \frac{Z_{2}(x)-Z_{1}(x)\left(W_{i}-x\right)}{Z_{0}(x) Z_{2}(x)-Z_{1}^{2}(x)}+O\left(\frac{(\log n)^{1+\gamma}}{\left(n h_{n}\right)^{\beta}}\right) \\
= & \frac{1}{n h_{n} f_{X}(x)} \sum_{i=1}^{n} g\left(X_{i}\right) K\left(\frac{W_{i}-x}{h_{n}}\right) \frac{v_{2}-v_{1} \frac{W_{i}-x}{h_{n}}}{v_{0} v_{2}-v_{1}^{2}}+O\left(h_{n}+\sigma_{n}+\frac{(\log n)^{1+\gamma}}{\left(n h_{n}\right)^{\beta}}\right) \\
= & \frac{1}{n h_{n} f_{X}(x)}\left[\sum_{X_{i}<x_{s}}+\sum_{X_{i} \geq x_{s}}\right] g\left(X_{i}\right) K\left(\frac{W_{i}-x}{h_{n}}\right) \frac{v_{2}-v_{1} \frac{W_{i}-x}{h_{n}}}{v_{0} v_{2}-v_{1}^{2}}+ \\
& O\left(h_{n}+\sigma_{n}+\frac{(\log n)^{1+\gamma}}{\left(n h_{n}\right)^{\beta}}\right) \\
= & \frac{1}{n h_{n} f_{X}(x)} \sum_{X_{i}<x_{s}}\left[g_{-}\left(x_{s}\right)+g^{\prime}\left(\theta_{i}^{-}\right)\left(W_{i}-x-\sigma_{n} U_{i}\right)\right] K\left(\frac{W_{i}-x}{h_{n}}\right) \frac{v_{2}-v_{1} \frac{W_{i}-x}{h_{n}}}{v_{0} v_{2}-v_{1}^{2}}
\end{aligned}
$$




$$
\begin{aligned}
& +\frac{1}{n h_{n} f_{X}(x)} \sum_{X_{i} \geq x_{s}}\left[g_{+}\left(x_{s}\right)+g^{\prime}\left(\theta_{i}^{+}\right)\left(W_{i}-x-\sigma_{n} U_{i}\right)\right] K\left(\frac{W_{i}-x}{h_{n}}\right) \frac{v_{2}-v_{1} \frac{W_{i}-x}{h_{n}}}{v_{0} v_{2}-v_{1}^{2}} \\
& +O\left(h_{n}+\sigma_{n}+\frac{(\log n)^{1+\gamma}}{\left(n h_{n}\right)^{\beta}}\right) \\
& =\frac{g_{-}\left(x_{s}\right)}{n h_{n} f_{X}(x)} \sum_{i=1}^{n} \mathbb{1}_{\left\{X_{i}-x<s h_{n}\right\}} K\left(\frac{W_{i}-x}{h_{n}}\right) \frac{v_{2}-v_{1} \frac{W_{i}-x}{h_{n}}}{v_{0} v_{2}-v_{1}^{2}} \\
& +\frac{g_{+}\left(x_{s}\right)}{n h_{n} f_{X}(x)} \sum_{i=1}^{n} \mathbb{1}_{\left\{X_{i}-x \geq s h_{n}\right\}} K\left(\frac{W_{i}-x}{h_{n}}\right) \frac{v_{2}-v_{1} \frac{W_{i}-x}{h_{n}}}{v_{0} v_{2}-v_{1}^{2}} \\
& +O\left(h_{n}+\sigma_{n}+\frac{(\log n)^{1+\gamma}}{\left(n h_{n}\right)^{\beta}}\right) \\
& =\frac{g_{-}\left(x_{s}\right)}{f_{X}(x)} \int_{-\infty}^{\infty} \int_{-\infty}^{\infty} \mathbb{1}_{\left\{w-u \frac{\sigma_{n}}{h_{n}}<s\right\}} K(w) \frac{v_{2}-v_{1} w}{v_{0} v_{2}-v_{1}^{2}} f_{X}\left(x+w h_{n}-\sigma_{n} u\right) f_{U}(u) d w d u \\
& +\frac{g_{+}\left(x_{s}\right)}{f_{X}(x)} \int_{-\infty}^{\infty} \int_{-\infty}^{\infty} \mathbb{1}_{\left\{w-u \frac{\sigma_{n}}{h_{n}} \geq s\right\}} K(w) \frac{v_{2}-v_{1} w}{v_{0} v_{2}-v_{1}^{2}} f_{X}\left(x+w h_{n}-\sigma_{n} u\right) f_{U}(u) d w d u \\
& +O\left(h_{n}+\sigma_{n}+\frac{(\log n)^{1+\gamma}}{\left(n h_{n}\right)^{\beta}}\right) \\
& =g_{-}\left(x_{s}\right) \int_{-\infty}^{\infty} f_{U}(u) \int_{-1}^{s+u \frac{\sigma_{n}}{h_{n}}} K(w) \frac{v_{2}-v_{1} w}{v_{0} v_{2}-v_{1}^{2}} d w d u+ \\
& g_{+}\left(x_{s}\right) \int_{-\infty}^{\infty} f_{U}(u) \int_{s+u \frac{\sigma_{n}}{h_{n}}}^{1} K(w) \frac{v_{2}-v_{1} w}{v_{2} v_{0}-v_{1}^{2}} d w d u \\
& +O\left(h_{n}+\sigma_{n}+\frac{(\log n)^{1+\gamma}}{\left(n h_{n}\right)^{\beta}}\right) \\
& =g_{-}\left(x_{s}\right)\left(\int_{-\infty}^{-(1+s) \frac{h_{n}}{\sigma_{n}}}+\int_{-(1+s) \frac{h_{n}}{\sigma_{n}}}^{(1-s) \frac{h_{n}}{\sigma_{n}}}+\int_{(1-s) \frac{h_{n}}{\sigma_{n}}}^{\infty}\right) f_{U}(u) \int_{-1}^{s+u \frac{\sigma_{n}}{h_{n}}} K(w) d w d u \\
& +g_{+}\left(x_{s}\right)\left(\int_{-\infty}^{-(1+s) \frac{h_{n}}{\sigma_{n}}}+\int_{-(1+s) \frac{h_{n}}{\sigma_{n}}}^{(1-s) \frac{h_{n}}{\sigma_{n}}}+\int_{(1-s) \frac{h_{n}}{\sigma_{n}}}^{\infty} f_{U}(u) \int_{-1}^{s+u \frac{\sigma_{n}}{h_{n}}} K(w) d w d u\right. \\
& =g_{-}\left(x_{s}\right) \operatorname{pr}\left(U_{1}>0\right)+g_{+}\left(x_{s}\right) \operatorname{pr}\left(U_{1} \leq 0\right)+O\left(h_{n}+\sigma_{n}+\frac{h_{n}}{\sigma_{n}}+\frac{(\log n)^{1+\gamma}}{\left(n h_{n}\right)^{\beta}}\right) \\
& =\frac{1}{2}\left[g_{-}\left(x_{s}\right)+g_{+}\left(x_{s}\right)\right]+O\left(h_{n}+\sigma_{n}+\frac{h_{n}}{\sigma_{n}}+\frac{(\log n)^{1+\gamma}}{\left(n h_{n}\right)^{\beta}}\right) \quad \text { a.s. }
\end{aligned}
$$


Next, we calculate $\widehat{b}_{n}(x)$.

$$
\begin{aligned}
& \widehat{b}_{n}(x) \\
& =\sum_{i=1}^{n} Y_{i} K\left(\frac{W_{i}-x}{h_{n}}\right) \frac{Z_{0}(x)\left(W_{i}-x\right)-Z_{1}(x)}{Z_{0}(x) Z_{2}(x)-Z_{1}^{2}(x)} \\
& =\sum_{i=1}^{n} g\left(X_{i}\right) K\left(\frac{W_{i}-x}{h_{n}}\right) \frac{Z_{0}(x)\left(W_{i}-x\right)-Z_{1}(x)}{Z_{0}(x) Z_{2}(x)-Z_{1}^{2}(x)}+\frac{1}{h_{n}} O\left(\frac{(\log n)^{1+\gamma}}{\left(n h_{n}\right)^{\beta}}\right) \\
& =\frac{1}{n h_{n}^{2} f_{X}(x)} \sum_{i=1}^{n} g\left(X_{i}\right) K\left(\frac{W_{i}-x}{h_{n}}\right) \frac{v_{0} \frac{W_{i}-x}{h_{0}}-v_{1}}{v_{0} v_{2}-v_{1}^{2}}+\frac{1}{h_{n}} O\left(h_{n}+\sigma_{n}+\frac{(\log n)^{1+\gamma}}{\left(n h_{n}\right)^{\beta}}\right) \\
& =\frac{1}{n h_{n}^{2} f_{X}(x)} \sum_{X_{i}<x_{s}} g\left(X_{i}\right) K\left(\frac{W_{i}-x}{h_{n}}\right) \frac{v_{0} \frac{W_{i}-x}{h_{n}}-v_{1}}{v_{0} v_{2}-v_{1}^{2}} \\
& +\frac{1}{n h_{n}^{2} f_{X}(x)} \sum_{X_{i} \geq x_{s}} g\left(X_{i}\right) K\left(\frac{W_{i}-x}{h_{n}}\right) \frac{v_{0} \frac{W_{i}-x}{h_{n}}-v_{1}}{v_{0} v_{2}-v_{1}^{2}}+\frac{1}{h_{n}} O\left(h_{n}+\sigma_{n}+\frac{(\log n)^{1+\gamma}}{\left(n h_{n}\right)^{\beta}}\right) \\
& =\frac{g_{-}\left(x_{s}\right)}{f_{X}(x) h_{n}} \int_{-\infty}^{\infty} \int_{-\infty}^{\infty} \mathbb{1}_{\left\{w-u \frac{\sigma_{n}}{h_{n}}<s\right\}} K(w) \frac{v_{0} w-v_{1}}{v_{0} v_{2}-v_{1}^{2}} f_{X}\left(x+w h_{n}-\sigma_{n} u\right) f_{U}(u) d w d u \\
& +\frac{g_{+}\left(x_{s}\right)}{f_{X}(x) h_{n}} \int_{-\infty}^{\infty} \int_{-\infty}^{\infty} \mathbb{1}_{\left\{w-u \frac{\sigma_{n}}{h_{n}} \geq s\right\}} K(w) \frac{v_{0} w-v_{1}}{v_{0} v_{2}-v_{1}^{2}} f_{X}\left(x+w h_{n}-\sigma_{n} u\right) f_{U}(u) d w d u \\
& +\frac{1}{h_{n}} O\left(h_{n}+\sigma_{n}+\frac{(\log n)^{1+\gamma}}{\left(n h_{n}\right)^{\beta}}\right) \\
& =\frac{g_{-}\left(x_{s}\right)}{h_{n}} \int_{-\infty}^{\infty} f_{U}(u) \int_{-1}^{s+u \frac{\sigma_{n}}{h_{n}}} K(w) \frac{v_{0} w-v_{1}}{v_{0} v_{2}-v_{1}^{2}} d w d u \\
& +\frac{g_{+}\left(x_{s}\right)}{h_{n}} \int_{-\infty}^{\infty} \int_{s+u \frac{\sigma_{n}}{h_{n}}}^{1} K(w) \frac{v_{0} w-v_{1}}{v_{0} v_{2}-v_{1}^{2}} d w d u+\frac{1}{h_{n}} O\left(h_{n}+\sigma_{n}+\frac{(\log n)^{1+\gamma}}{\left(n h_{n}\right)^{\beta}}\right) \\
& =\frac{1}{h_{n}} O\left(\frac{h_{n}}{\sigma_{n}}+h_{n}+\sigma_{n}+\frac{(\log n)^{1+\gamma}}{\left(n h_{n}\right)^{\beta}}\right) \quad \text { a.s. }
\end{aligned}
$$

Next,

$$
\begin{aligned}
& \frac{1}{n h_{n}} \sum_{i=1}^{n}\left[g\left(X_{i}\right)+\varepsilon_{i}-\widehat{a}_{n}(x)-\widehat{b}_{n}(x)\left(W_{i}-x\right)\right]^{2} K\left(\frac{W_{i}-x}{h_{n}}\right) \\
= & \frac{\tau^{2}}{n h_{n}} \sum_{i=1}^{n} K\left(\frac{W_{i}-x}{h_{n}}\right)+\frac{1}{n h_{n}} \sum_{i=1}^{n}\left(\varepsilon_{i}^{2}-\tau^{2}\right) K\left(\frac{W_{i}-x}{h_{n}}\right) \\
& +\frac{2}{n h_{n}} \sum_{i=1}^{n} \varepsilon_{i}\left[g\left(X_{i}\right)-\widehat{a}_{n}(x)-\widehat{b}_{n}(x)\left(W_{i}-x\right)\right] K\left(\frac{W_{i}-x}{h_{n}}\right)
\end{aligned}
$$




$$
+\frac{1}{n h_{n}} \sum_{i=1}^{n}\left[g\left(X_{i}\right)-\widehat{a}_{n}(x)-\widehat{b}_{n}(x)\left(W_{i}-x\right)\right]^{2} K\left(\frac{W_{i}-x}{h_{n}}\right) .
$$

By Lemma 2, we have that

$$
\frac{(\mathrm{S} .38)}{\frac{1}{n h_{n}} \sum_{i=1}^{n} K\left(\frac{W_{i}-x}{h_{n}}\right)}=\tau^{2}+O\left(\frac{(\log n)^{1+\gamma}}{\left(n h_{n}\right)^{\beta}}\right) \text { a.s. }
$$

Also,

$$
\begin{aligned}
(\mathrm{S} .39)= & \frac{2}{n h_{n}} \sum_{i=1}^{n} \varepsilon_{i}\left(g\left(X_{i}\right)-\widehat{a}_{n}(x)+h_{n} \widehat{b}_{n}(x) \frac{W_{i}-x}{h_{n}}\right) K\left(\frac{W_{i}-x}{h_{n}}\right) \\
= & \frac{2}{n h_{n}} \sum_{i=1}^{n} \varepsilon_{i}\left(g\left(X_{i}\right)-\widehat{a}_{n}(x)\right) K\left(\frac{W_{i}-x}{h_{n}}\right)+O\left(\frac{h_{n}}{\sigma_{n}}+h_{n}+\sigma_{n}+\frac{(\log n)^{1+\gamma}}{\left(n h_{n}\right)^{\beta}}\right) \\
= & \frac{2}{n h_{n}} \sum_{X_{i}<x_{s}} \varepsilon_{i}\left[g_{-}\left(x_{s}\right)+g^{\prime}\left(\theta_{i}^{-}\right)\left(W_{i}-\sigma_{n} U_{i}-x+x-x_{s}\right)-\widehat{a}_{n}(x)\right] K\left(\frac{W_{i}-x}{h_{n}}\right) \\
& +\frac{2}{n h_{n}} \sum_{X_{i} \geq x_{s}} \varepsilon_{i}\left[g_{+}\left(x_{s}\right)+g^{\prime}\left(\theta_{i}^{+}\right)\left(W_{i}-\sigma_{n} U_{i}-x+x-x_{s}\right)-\widehat{a}_{n}(x)\right] K\left(\frac{W_{i}-x}{h_{n}}\right) \\
& +O\left(\frac{h_{n}}{\sigma_{n}}+h_{n}+\sigma_{n}+\frac{(\log n)^{1+\gamma}}{\left(n h_{n}\right)^{\beta}}\right) \\
= & \frac{1}{n h_{n}} \sum_{X_{i}<x_{s}}\left[g_{-}\left(x_{s}\right)-g_{+}\left(x_{s}\right)\right] \varepsilon_{i} K\left(\frac{W_{i}-x}{h_{n}}\right)+O\left(\frac{h_{n}}{\sigma_{n}}+h_{n}+\sigma_{n}+\frac{(\log n)^{1+\gamma}}{\left(n h_{n}\right)^{\beta}}\right) \\
& +\frac{1}{n h_{n}} \sum_{X_{i} \geq x_{s}}\left[g_{+}\left(x_{s}\right)-g_{-}\left(x_{s}\right)\right] \varepsilon_{i} K\left(\frac{W_{i}-x}{h_{n}}\right) \\
= & O\left(\frac{h_{n}}{\sigma_{n}}+h_{n}+\sigma_{n}+\frac{(\log n)^{1+\gamma}}{\left(n h_{n}\right)^{\beta}}\right) a . s .
\end{aligned}
$$

And

$$
\begin{aligned}
(\mathrm{S} .40)= & \frac{1}{n h_{n}} \sum_{i=1}^{n}\left[g\left(X_{i}\right)-\widehat{a}_{n}(x)-h_{n} \widehat{b}_{n}(x) \frac{W_{i}-x}{h_{n}}\right]^{2} K\left(\frac{W_{i}-x}{h_{n}}\right) \\
= & \frac{1}{n h_{n}} \sum_{i=1}^{n}\left[g\left(X_{i}\right)-\widehat{a}_{n}(x)\right]^{2} K\left(\frac{W_{i}-x}{h_{n}}\right)+O\left(\frac{h_{n}}{\sigma_{n}}+h_{n}+\sigma_{n}+\frac{(\log n)^{1+\gamma}}{\left(n h_{n}\right)^{\beta}}\right) \\
= & \frac{1}{4 n h_{n}} \sum_{X_{i}<x_{s}}\left[g_{-}\left(x_{s}\right)-g_{+}\left(x_{s}\right)\right]^{2} K\left(\frac{W_{i}-x}{h_{n}}\right)+O\left(\frac{h_{n}}{\sigma_{n}}+h_{n}+\sigma_{n}+\frac{(\log n)^{1+\gamma}}{\left(n h_{n}\right)^{\beta}}\right) \\
& +\frac{1}{4 n h_{n}} \sum_{X_{i} \geq x_{s}}\left[g_{+}\left(x_{s}\right)-g_{-}\left(x_{s}\right)\right]^{2} K\left(\frac{W_{i}-x}{h_{n}}\right)
\end{aligned}
$$




$$
=\frac{d_{s}^{2}}{4 n h_{n}} \sum_{i=1}^{n} K\left(\frac{W_{i}-x}{h_{n}}\right)+O\left(\frac{h_{n}}{\sigma_{n}}+h_{n}+\sigma_{n}+\frac{(\log n)^{1+\gamma}}{\left(n h_{n}\right)^{\beta}}\right) \text { a.s. }
$$

Therefore,

$$
\operatorname{WRMS}_{n}(x)=\tau^{2}+\frac{d_{s}^{2}}{4}+O\left(\frac{h_{n}}{\sigma_{n}}+\sigma_{n}+\frac{(\log n)^{1+\gamma}}{\left(n h_{n}\right)^{\beta}}\right) \text { a.s. }
$$

(iii) Now we calculate $\widehat{a}_{n}(x)$ when $\sigma_{n} / h_{n}=O(1)$.

$$
\begin{aligned}
\widehat{a}_{n}(x)= & \sum_{i=1}^{n} Y_{i} K\left(\frac{W_{i}-x}{h_{n}}\right) \frac{Z_{2}(x)-Z_{1}(x)\left(W_{i}-x\right)}{Z_{0}(x) Z_{2}(x)-Z_{1}(x)^{2}} \\
= & \frac{1}{n h_{n} f_{X}(x)} \sum_{i=1}^{n} g\left(X_{i}\right) K\left(\frac{W_{i}-x}{h_{n}}\right) \frac{v_{2}-v_{1} \frac{W_{i}-x}{h_{n}}}{v_{0} v_{2}-v_{1}^{2}}+O\left(h_{n}+\sigma_{n}+\frac{(\log n)^{1+\gamma}}{\left(n h_{n}\right)^{\beta}}\right) \\
= & g_{-}\left(x_{s}\right) \int_{-\infty}^{\infty} f_{U}(u) \int_{-1}^{s+u \frac{\sigma_{n}}{h_{n}}} K(w) \frac{v_{2}-v_{1} w}{v_{0} v_{2}-v_{1}^{2}} d w d u+O\left(h_{n}+\sigma_{n}+\frac{(\log n)^{1+\gamma}}{\left(n h_{n}\right)^{\beta}}\right) \\
& +g_{+}\left(x_{s}\right) \int_{-\infty}^{\infty} f_{U}(u) \int_{s+u \frac{\sigma_{n}}{h_{n}}}^{1} K(w) \frac{v_{2}-v_{1} w}{v_{0} v_{2}-v_{1}^{2}} d w d u \\
= & g_{-}\left(x_{s}\right)+d_{s} \int_{-\infty}^{\infty} f_{U}(u) \int_{s+u \frac{\sigma_{n}}{h_{n}}}^{1} K(w) \frac{v_{2}-v_{1} w}{v_{0} v_{2}-v_{1}^{2}} d w d u+O\left(h_{n}+\sigma_{n}+\frac{(\log n)^{1+\gamma}}{\left(n h_{n}\right)^{\beta}}\right) \text { a.s. }
\end{aligned}
$$

Next,

$$
\begin{aligned}
\widehat{b}_{n}(x)= & \sum_{i=1}^{n} g\left(X_{i}\right) K\left(\frac{W_{i}-x}{h_{n}}\right) \frac{Z_{0}(x)\left(W_{i}-x\right)-Z_{1}(x)}{Z_{0}(x) Z_{2}(x)-Z_{1}^{2}(x)}+\frac{1}{h_{n}} O\left(\frac{(\log n)^{1+\gamma}}{\left(n h_{n}\right)^{\beta}}\right) \\
= & \frac{1}{f_{X}(x) n h_{n}^{2}} \sum_{i=1}^{n} g\left(X_{i}\right) K\left(\frac{W_{i}-x}{h_{n}}\right) \frac{v_{0} \frac{W_{i}-x}{h_{n}}-v_{1}}{v_{0} v_{2}-v_{1}^{2}}+\frac{1}{h_{n}} O\left(h_{n}+\sigma_{n}+\frac{(\log n)^{1+\gamma}}{\left(n h_{n}\right)^{\beta}}\right) \\
= & \frac{g_{-}\left(x_{s}\right)}{h_{n}} \int_{-\infty}^{\infty} f_{U}(u) \int_{-1}^{s+u \frac{\sigma_{n}}{h_{n}}} K(w) \frac{v_{0} w-v_{1}}{v_{0} v_{2}-v_{1}^{2}} d w d u+\frac{1}{h_{n}} O\left(h_{n}+\sigma_{n}+\frac{(\log n)^{1+\gamma}}{\left(n h_{n}\right)^{\beta}}\right) \\
& +\frac{g_{+}\left(x_{s}\right)}{h_{n}} \int_{-\infty}^{\infty} f_{U}(u) \int_{-\infty}^{1} K(w) \frac{v_{0} w-v_{1}}{v_{0} v_{2}-v_{1}^{2}} d w d u \\
= & \frac{d_{s}}{h_{n}} \int_{-\infty}^{\infty} f_{U}(u) \int_{s+u \frac{\sigma_{n}}{h_{n}}}^{1} K(w) \frac{v_{0} w-v_{1}}{v_{0} v_{2}-v_{1}^{2}} d w d u+\frac{1}{h_{n}} O\left(h_{n}+\sigma_{n}+\frac{(\log n)^{1+\gamma}}{\left(n h_{n}\right)^{\beta}}\right) \\
= & \frac{d_{s}}{h_{n}} \int_{-\infty}^{\infty} f_{U}(u) \int_{s+u \frac{\sigma_{n}}{h_{n}}}^{1} K(w) \frac{w}{v_{2}} d w d u+\frac{1}{h_{n}} O\left(h_{n}+\sigma_{n}+\frac{(\log n)^{1+\gamma}}{\left(n h_{n}\right)^{\beta}}\right)^{a . s .}
\end{aligned}
$$


It remains to calculate $\mathrm{WRMS}_{n}(x)$. By the arguments similar to those in (ii), we have

$$
\frac{\frac{1}{n h_{n}} \sum_{i=1}^{n} \varepsilon_{i}^{2} K\left(\frac{W_{i}-x}{h_{n}}\right)}{\frac{1}{n h_{n}} \sum_{i=1}^{n} K\left(\frac{W_{i}-x}{h_{n}}\right)}=\tau^{2}+O\left(\frac{(\log n)^{1+\gamma}}{\left(n h_{n}\right)^{\beta}}\right) \text { a.s. }
$$

Also, by Lemma 2,

$$
\begin{aligned}
& \frac{2}{n h_{n}} \sum_{i=1}^{n} \varepsilon_{i}\left[g\left(X_{i}\right)-\widehat{a}_{n}(x)-\widehat{b}_{n}(x)\left(W_{i}-x\right)\right] K\left(\frac{W_{i}-x}{h_{n}}\right) \\
= & \frac{2}{n h_{n}} \sum_{X_{i}<x_{s}} \varepsilon_{i}\left[g_{-}\left(x_{s}\right)-\widehat{a}_{n}(x)\right] K\left(\frac{W_{i}-x}{h_{n}}\right) \\
& +\frac{2}{n h_{n}} \sum_{X_{i} \geq x_{s}} \varepsilon_{i}\left[g_{+}\left(x_{s}\right)-\widehat{a}_{n}(x)\right] K\left(\frac{W_{i}-x}{h_{n}}\right) \\
& +\frac{2 h_{n} \widehat{b}_{n}(x)}{n h_{n}} \sum_{i=1}^{n} \varepsilon_{i} \frac{W_{i}-x}{h_{n}} K\left(\frac{W_{i}-x}{h_{n}}\right)+O\left(h_{n}+\sigma_{n}\right) \\
= & O\left(h_{n}+\sigma_{n}+\frac{(\log n)^{1+\gamma}}{\left(n h_{n}\right)^{\beta}}\right) \text { a.s. }
\end{aligned}
$$

Next,

$$
\begin{aligned}
& \frac{1}{n h_{n}} \sum_{i=1}^{n}\left[g\left(X_{i}\right)-\widehat{a}_{n}(x)-\widehat{b}_{n}(x)\left(W_{i}-x\right)\right]^{2} K\left(\frac{W_{i}-x}{h_{n}}\right) \\
= & \frac{d_{s}^{2}}{n h_{n}} \sum_{X_{i}<x_{s}}\left[\int_{-\infty}^{\infty} f_{U}(u) \int_{s+u \frac{\sigma_{n}}{h_{n}}}^{1} K(z) \frac{v_{2}-v_{1} z}{v_{0} v_{2}-v_{1}^{2}} d z d u+\int_{-\infty}^{\infty} f_{U}(u) \int_{s+u \sigma_{n}}^{1} K(z) \frac{v_{0} z-v_{1}}{v_{0} v_{2}-v_{1}^{2}} d z d u \frac{W_{i}-x}{h_{n}}\right]^{2} \\
& \cdot K\left(\frac{W_{i}-x}{h_{n}}\right)+O\left(h_{n}+\sigma_{n}+\frac{(\log n)^{1+\gamma}}{\left(n h_{n}\right)^{\beta}}\right) \\
& +\frac{d_{s}^{2}}{n h_{n}} \sum_{X_{i} \geq x_{s}}\left[\int_{-\infty}^{\infty} f_{U}(u) \int_{-1}^{s+u \frac{\sigma_{n}}{h_{n}}} K(z) \frac{v_{2}-v_{1} z}{v_{0} v_{2}-v_{1}^{2}} d z d u-\int_{-\infty}^{\infty} f_{U}(u) \int_{s+u \frac{\sigma_{n}}{h_{n}}}^{1} K(z) \frac{v_{0} z-v_{1}}{v_{0} v_{2}-v_{1}^{2}} d z d u \frac{W_{i}-x}{h_{n}}\right]^{2} \\
& \cdot K\left(\frac{W_{i}-x}{h_{n}}\right) \int_{-\infty}^{1} \int_{s}^{2} K(z) \frac{v_{2}-v_{1} z}{v_{0} v_{2}-v_{1}^{2}} d z d u^{\prime} \\
& d_{s}^{2} f_{X}(x) \int_{-\infty}^{\infty} f_{U}(u) \int_{-1}^{s+u \frac{\sigma_{n}}{h_{n}}}\left[\int_{-\infty}^{\infty} f_{U}\left(u^{\prime}\right)\right. \\
& \left.+\int_{-\infty}^{\infty} f_{U}\left(u^{\prime}\right) \int_{s+u^{\prime} \frac{\sigma_{n}}{h_{n}}}^{1} K(z) \frac{v_{0} z-v_{1}}{v_{0} v_{2}-v_{1}^{2}} d z d u^{\prime} \cdot w\right]^{2} K(w) d w d u+O\left(h_{n}+\sigma_{n}+\frac{(\log n)^{1+\gamma}}{\left(n h_{n}\right)^{\beta}}\right)
\end{aligned}
$$




$$
\begin{aligned}
& +d_{s}^{2} f_{X}(x) \int_{-\infty}^{\infty} f_{U}(u) \int_{s+u \frac{\sigma_{n}}{h_{n}}}^{1}\left[\int_{-\infty}^{\infty} f_{U}\left(u^{\prime}\right) \int_{-1}^{s+u^{\prime} \frac{\sigma_{n}}{h_{n}}} K(z) \frac{v_{2}-v_{1} z}{v_{0} v_{2}-v_{1}^{2}} d z d u^{\prime}\right. \\
& \left.-\int_{-\infty}^{\infty} f_{U}\left(u^{\prime}\right) \int_{s+u^{\prime} \frac{\sigma_{n}}{h_{n}}}^{1} K(z) \frac{v_{0} z-v_{1}}{v_{0} v_{2}-v_{1}^{2}} d z d u^{\prime} \cdot w\right]^{2} K(w) d w d u \\
& =d_{s}^{2} f_{X}(x) \int_{-\infty}^{\infty} f_{U}(u) \int_{-1}^{s+u \frac{\sigma_{n}}{h_{n}}}\left[\int_{-\infty}^{\infty} f_{U}\left(u^{\prime}\right) \int_{s+u^{\prime} \frac{\sigma_{n}}{h_{n}}}^{1} K(z) d z d u^{\prime}+\int_{-\infty}^{\infty} f_{U}\left(u^{\prime}\right) \int_{s+u^{\prime} \frac{\sigma_{n}}{h_{n}}}^{1} K(z) \frac{z}{v_{2}} d z d u^{\prime} \cdot w\right]^{2} \\
& \cdot K(w) d w d u+O\left(h_{n}+\sigma_{n}+\frac{(\log n)^{1+\gamma}}{\left(n h_{n}\right)^{\beta}}\right) \\
& +d_{s}^{2} f_{X}(x) \int_{-\infty}^{\infty} f_{U}(u) \int_{s+u \frac{\sigma_{n}}{h_{n}}}^{1}\left[\int_{-\infty}^{\infty} f_{U}\left(u^{\prime}\right) \int_{-1}^{s+u^{\prime} \frac{\sigma_{n}}{h_{n}}} K(z) d z d u^{\prime}-\int_{-\infty}^{\infty} f_{U}\left(u^{\prime}\right) \int_{s+u^{\prime} \frac{\sigma_{n}}{h_{n}}}^{1} K(z) \frac{z}{v_{2}} d z d u^{\prime} \cdot w\right]^{2} \\
& \cdot K(w) d w d u \\
& =d_{s}^{2} f_{X}(x) C_{n}(s)+O\left(h_{n}+\sigma_{n}+\frac{(\log n)^{1+\gamma}}{\left(n h_{n}\right)^{\beta}}\right) \quad \text { a.s. }
\end{aligned}
$$

where $C_{n}(s)$ is a sequence of positive numbers that are bounded away from zero and bounded from above. Therefore,

$$
\mathrm{WRMS}_{n}(x)=\tau^{2}+d_{s}^{2} C_{n}(s)+O\left(h_{n}+\sigma_{n}+\frac{(\log n)^{1+\gamma}}{\left(n h_{n}\right)^{\beta}}\right) \quad \text { a.s. }
$$

The proof of Lemma 4 is completed.

\section{Proof of Theorem 1}

Proof. Combining the condition that $u_{n}=\tau^{2}+\delta_{n}, \delta_{n}=o(1),\left[h_{n}^{2}+\log (n)^{1+\gamma} /\left(n h_{n}\right)^{\beta}+\right.$ $\left.\sigma_{n}^{2}\right] / \delta_{n}=o(1)$, Lemma 3 and Lemma 4 leads to that, when $n$ is sufficiently large, the clustering procedure (6)-(8) is performed only for design points near jump locations. Then, by (S.17), Theorem is valid for $x$ in continuity region. In the case when $x$ is near a jump location (i.e., $x_{s}=x+s \cdot h_{n}^{\prime}$ with $s \in(-1,1)$ ), we claim that, when $n$ is sufficiently large, the clustering procedure (6)-(8) separates the observations in $G\left(x ; h_{n}^{\prime}\right)$ into two groups, where 
one group of observations have $X$ values smaller than $x_{s}$ and the other group of observations have $X$ values larger than or equal to $x_{s}$ (i.e., the misclassification rate tends to 0 as $n \rightarrow \infty$.). To prove this claim, by the strong law of large numbers, we have

$$
\begin{aligned}
\left|\bar{W}_{l}-\bar{W}_{r}\right|=o(1) \text { a.s. } & \left|\bar{Y}_{l}-\bar{Y}_{r}\right|=d_{s}+o(1) \text { a.s. } \\
\operatorname{WRMS}_{l}(x)=\tau^{2}+o(1) \text { a.s. } & \operatorname{WRMS}_{r}(x)=\tau^{2}+o(1) \text { a.s. }
\end{aligned}
$$

when the misclassification rate tends to 0 as $n \rightarrow 0$. Suppose that the clustering procedure (6)-(8) has a misclassification rate bounded from below by a positive constant. Then we have

$$
\left|\bar{W}_{l}-\bar{W}_{r}\right|=o(1) \text { a.s. } \quad\left|\bar{Y}_{l}-\bar{Y}_{r}\right|=d_{s}-\eta+o(1) \text { a.s. }
$$

for some $\eta>0$. Also, we have, by arguments similar to those in Lemma 4,

$$
\mathrm{WRMS}_{l}(x)=\tau^{2}+Q_{1}+o(1) \text { a.s. }, \quad \operatorname{WRMS}_{r}(x)=\tau^{2}+Q_{2}+o(1) \text { a.s. }
$$

where $Q_{1}$ and $Q_{2}$ are some positive quantities. Therefore, this contradicts the definition of the proposed clustering procedure which maximizes the separation measure defined in (8) and hence the claim is true.

Next, let

$$
\begin{aligned}
& \widetilde{G}_{l}\left(x ; h_{n}^{\prime}\right)=\left\{\left(W_{i}, Y_{i}\right) \in G\left(x ; h_{n}^{\prime}\right): X_{i} \leq x_{s}, i=1, \cdots, n\right\}, \\
& \widetilde{G}_{r}\left(x ; h_{n}^{\prime}\right)=\left\{\left(W_{i}, Y_{i}\right) \in G\left(x ; h_{n}^{\prime}\right): X_{i}>x_{s}, i=1, \cdots, n\right\} .
\end{aligned}
$$

Define

$$
\begin{aligned}
\left|\widetilde{G}_{r}\left(x ; h_{n}^{\prime}\right)\right| & =\frac{1}{n h_{n}^{\prime}} \sum_{i=1}^{n} \mathbb{1}_{\left\{X_{i} \geq x_{s}\right\}} \mathbb{1}_{\left\{x-h_{n}^{\prime}<X_{i}+\sigma_{n} U_{i}<x+h_{n}^{\prime}\right\}} . \\
\left|\widetilde{G}_{l}\left(x ; h_{n}^{\prime}\right)\right| & =\frac{1}{n h_{n}^{\prime}} \sum_{i=1}^{n} \mathbb{1}_{\left\{X_{i}<x_{s}\right\}} \mathbb{1}_{\left\{x-h_{n}^{\prime}<X_{i}+\sigma_{n} U_{i}<x+h_{n}^{\prime}\right\}} .
\end{aligned}
$$

Without loss of generality, assume that $s \in(0,1)$. Then, it remains to show that $\left|\widetilde{G}_{l}\left(x ; h_{n}^{\prime}\right)\right|>$ $\left|\widetilde{G}_{r}\left(x ; h_{n}^{\prime}\right)\right|$ a.s.. By arguments similar to those in Lemma 2 , it can be verified that

$$
|| \widetilde{G}_{j}\left(x ; h_{n}^{\prime}\right)\left|-\mathrm{E}\left\{\mid \widetilde{G}_{j}\left(x ; h_{n}^{\prime}\right)\right\}\right|=O\left(\left(\frac{\log n}{n h_{n}^{\prime}}\right)^{\frac{1}{2}}\right) \quad \text { a.s. }(j=l, r) .
$$


And it can be calculated that

$$
\begin{gathered}
\mathrm{E}\left\{\left|\widetilde{G}_{l}\left(x ; h_{n}^{\prime}\right)\right|\right\}=f_{X}\left(x_{s}\right)\left[2(1+s) \int_{0}^{(1-s) \frac{h_{n}^{\prime}}{\sigma_{n}}} f_{U}(u) d u+(1+s) \int_{(1-s) \frac{h_{n}^{\prime}}{\sigma_{n}}}^{(1+s) \frac{h_{n}^{\prime}}{\sigma_{n}}} f_{U}(u) d u\right. \\
\left.+2 \int_{(1-s) \frac{h_{n}^{\prime}}{\sigma_{n}}}^{\infty} f_{U}(u) d u-\frac{\sigma_{n}}{h_{n}^{\prime}} \int_{(1-s) \frac{h_{n}^{\prime}}{\sigma_{n}}}^{(1+s) \frac{h_{n}^{\prime}}{\sigma_{n}}} u f_{U}(u) d u\right]+O\left(h_{n}^{\prime}+\sigma_{n}\right) . \\
\mathrm{E}\left\{\left|\widetilde{G}_{r}\left(x ; h_{n}^{\prime}\right)\right|\right\}=f_{X}\left(x_{s}\right)\left[2(1-s) \int_{0}^{(1-s) \frac{h_{n}^{\prime}}{\sigma_{n}}} f_{U}(u) d u+(1-s) \int_{(1+s) \frac{h_{n}^{\prime}}{\sigma_{n}}}^{(1-s) \frac{h_{n}^{\prime}}{\sigma_{n}}} f_{U}(u) d u\right. \\
\left.+2 \int_{(1+s) \frac{h_{n}^{\prime}}{\sigma_{n}}}^{\infty} f_{U}(u) d u+\frac{\sigma_{n}}{h_{n}^{\prime}} \int_{(1-s) \frac{h_{n}^{\prime}}{\sigma_{n}}}^{(1+s) \frac{h_{n}^{\prime}}{\sigma_{n}}} u f_{U}(u) d u\right]+O\left(h_{n}^{\prime}+\sigma_{n}\right) .
\end{gathered}
$$

Thus, using symmetry of $f_{U}(\cdot)$,

$$
\begin{aligned}
& \mathrm{E}\left\{\left|\widetilde{G}_{l}\left(x ; h_{n}^{\prime}\right)\right|-\left|\widetilde{G}_{r}\left(x ; h_{n}^{\prime}\right)\right|\right\} \\
= & f_{X}\left(x_{s}\right)\left[4 s \int_{0}^{(1-s) \frac{h_{n}^{\prime}}{\sigma_{n}}} f_{U}(u) d u+2(1+s) \int_{(1-s) \frac{h_{n}^{\prime}}{\sigma_{n}}}^{(1+s) \frac{h_{n}^{\prime}}{\sigma_{n}}} f_{U}(u) d u-2 \frac{\sigma_{n}}{h_{n}^{\prime}} \int_{(1-s) \frac{h_{n}^{\prime}}{\sigma_{n}}}^{(1+s) \frac{h_{n}^{\prime}}{\sigma_{n}}} u f_{U}(u) d u\right]+ \\
& O\left(h_{n}^{\prime}+\sigma_{n}\right) .
\end{aligned}
$$

In cases when $\sigma_{n} / h_{n}^{\prime}=o(1)$, we have

$$
\left|\widetilde{G}_{l}\left(x ; h_{n}^{\prime}\right)\right|-\left|\widetilde{G}_{r}\left(x ; h_{n}^{\prime}\right)\right|=f_{X}\left(x_{s}\right) \cdot 2 s+O\left(\frac{\sigma_{n}}{h_{n}^{\prime}}+h_{n}^{\prime}+\left(\frac{\log n}{n h_{n}^{\prime}}\right)^{\frac{1}{2}}\right) \text { a.s. }
$$

In cases when $\sigma_{n} / h_{n}^{\prime} \rightarrow \infty$, we have

$$
\begin{aligned}
& \mathrm{E}\left\{\left|\widetilde{G}_{l}\left(x ; h_{n}^{\prime}\right)\right|-\left|\widetilde{G}_{r}\left(x ; h_{n}^{\prime}\right)\right|\right\} \\
= & f_{X}\left(x_{s}\right) f_{U}(0)\left[4 s(1-s) \frac{h_{n}^{\prime}}{\sigma_{n}}+4 s(1+s) \frac{h_{n}^{\prime}}{\sigma_{n}}-2 \frac{\sigma_{n}}{h_{n}^{\prime}} \int_{(1-s) \frac{h_{n}^{\prime}}{\sigma_{n}}}^{(1+s) \frac{h_{n}^{\prime}}{\sigma_{n}}} u d u\right]+ \\
& O\left(\left(\frac{h_{n}^{\prime}}{\sigma_{n}}\right)^{2}+h_{n}^{\prime}+\sigma_{n}\right)
\end{aligned}
$$




$$
=\frac{h_{n}^{\prime}}{\sigma_{n}}\left[f_{X}\left(x_{s}\right) f_{U}(0) \cdot 4 s+O\left(\frac{h_{n}^{\prime}}{\sigma_{n}}+\sigma_{n}+\frac{\sigma_{n}^{2}}{h_{n}^{\prime}}\right)\right] .
$$

And

$$
\begin{aligned}
& \left|\widetilde{G}_{l}\left(x ; h_{n}^{\prime}\right)\right|-\left|\widetilde{G}_{r}\left(x ; h_{n}^{\prime}\right)\right|= \\
& \frac{h_{n}^{\prime}}{\sigma_{n}}\left[f_{X}\left(x_{s}\right) f_{U}(0) \cdot 4 s+O\left(\frac{h_{n}^{\prime}}{\sigma_{n}}+\sigma_{n}+\frac{\sigma_{n}^{2}}{h_{n}^{\prime}}+\left(\frac{\sigma_{n}^{2} \log n}{n h_{n}^{\prime 3}}\right)^{\frac{1}{2}}\right)\right] \text { a.s. }
\end{aligned}
$$

In cases when $\sigma_{n} / h_{n}^{\prime}=O(1)$, we define

$$
H(\zeta)=4 s \int_{0}^{\frac{1-s}{\zeta}} f_{U}(u) d u+2(1+s) \int_{\frac{1-s}{\zeta}}^{\frac{1+s}{\zeta}} f_{U}(u) d u-2 \zeta \int_{\frac{1-s}{\zeta}}^{\frac{1+s}{\zeta}} u f_{U}(u) d u
$$

where $\zeta \in(0, \infty)$. It can be checked that $\lim _{\zeta \rightarrow 0} H(\zeta)=2 s, \lim _{\zeta \rightarrow \infty} H\left(\zeta_{n}\right)=0$, and

$$
H^{\prime}(\zeta)=-\int_{\frac{1-s}{\zeta_{n}}}^{\frac{1+s}{\theta_{n}}} u f_{U}(u) d u<0
$$

So, $H(\cdot)$ is a strictly decreasing function on $(0, \infty)$ with range $(0,2 s)$. Then, by $\sigma_{n} / h_{n}^{\prime}=$ $O(1)$, we have

$$
\begin{aligned}
\left|\widetilde{G}_{l}\left(x ; h_{n}^{\prime}\right)\right|-\left|\widetilde{G}_{r}\left(x ; h_{n}^{\prime}\right)\right| & =f_{X}\left(x_{s}\right) H\left(\frac{\sigma_{n}}{h_{n}^{\prime}}\right)+O\left(h_{n}^{\prime}+\sigma_{n}+\left(\frac{\log n}{n h_{n}^{\prime}}\right)^{\frac{1}{2}}\right) \\
& \geq f_{X}\left(x_{s}\right) H\left(C_{8}\right)+O\left(h_{n}^{\prime}+\sigma_{n}+\left(\frac{\log n}{n h_{n}^{\prime}}\right)^{\frac{1}{2}}\right) \quad \text { a.s., }
\end{aligned}
$$

where $C_{8}$ is some constant. By (S.41)-(S.43) and the fact that $f_{X}$ is positive on $(0,1)$, we conclude that $\left|\widetilde{G}_{l}\left(x ; h_{n}^{\prime}\right)\right|>\left|\widetilde{G}_{r}\left(x ; h_{n}^{\prime}\right)\right|$ a.s. when $n$ is sufficiently large and therefore, the proof of Theorem 1 is completed.

\section{References}

Butler, J. R. (2002). Policy change and private health insurance: Did the cheapest policy do the trick? Australian Health Review, 25(6):33-41. 
Carroll, R. J., Maca, J. D., and Ruppert, D. (1999). Nonparametric regression in the presence of measurement error. Biometrika, 86(3):541-554.

Carroll, R. J., Ruppert, D., Stefanski, L. A., and Crainiceanu, C. M. (2012). Measurement error in nonlinear models: a modern perspective. CRC press.

Comte, F. and Taupin, M.-L. (2007). Nonparametric estimation of the regression function in an errors-in-variables model. Statistica Sinica, 17:1065-1090.

Cook, J. R. and Stefanski, L. A. (1994). Simulation-extrapolation estimation in parametric measurement error models. Journal of the American Statistical Association, 89(428):13141328.

Cox, D. (1970). The Analysis of Binary Data. Chapman and Hall, London.

Delaigle, A. (2008). An alternative view of the deconvolution problem. Statistica Sinica, 18(3):1025-1045.

Delaigle, A. and Meister, A. (2007). Nonparametric regression estimation in the heteroscedastic errors-in-variables problem. Journal of the American Statistical Association, 102(480):1416-1426.

Eubank, R. and Speckman, P. (1994). Nonparametric estimation of functions with jump discontinuities. Lecture Notes-Monograph Series, pages 130-144.

Everitt, B. S., Landau, S., Leese, M., and Stahl, D. (2011). Cluster Analysis. John Wiley and Sons, Ltd., 5th edition.

Fan, J. and Masry, E. (1992). Multivariate regression estimation with errors-in-variables: asymptotic normality for mixing processes. Journal of multivariate analysis, 43(2):237271.

Fan, J. and Truong, Y. K. (1993). Nonparametric regression with errors in variables. The Annals of Statistics, 21(4):1900-1925. 
Frech, H. E., Hopkins, S., and MacDonald, G. (2003). The australian private health insurance boom: was it subsidies or liberalised regulation? Economic Papers: A journal of applied economics and policy, 22(1):58-64.

Gijbels, I. and Goderniaux, A.-C. (2004). Bandwidth selection for changepoint estimation in nonparametric regression. Technometrics, 46(1):76-86.

Gijbels, I., Hall, P., and Kneip, A. (1999). On the estimation of jump points in smooth curves. Annals of the Institute of Statistical Mathematics, 51(2):231-251.

Gijbels, I., Lambert, A., and Qiu, P. (2007). Jump-preserving regression and smoothing using local linear fitting: a compromise. Annals of the Institute of Statistical Mathematics, 59(2):235-272.

Hall, P. and Meister, A. (2007). A ridge-parameter approach to deconvolution. The Annals of Statistics, 35(4):1535-1558.

Hartigan, J. and Wong, M. (1979). Algorithm as 136: A k-means clustering algorithm. Journal of the Royal Statistical Society. Series C (Applied Statistics), 28(1):100-108.

Kang, Y., Gong, X., Gao, J., and Qiu, P. (2015). Jump detection in generalized error-invariables regression with an application to australian health tax policies. The Annals of Applied Statistics, 9:883-900.

Kang, Y. and Qiu, P. (2014). Jump detection in blurred regression surfaces. Technometrics, 56:539-550.

McDonald, J. A. and Owen, A. B. (1986). Smoothing with split linear fits. Technometrics, 28(3):195-208.

Müller, C. H. (2002). Robust estimators for estimating discontinuous functions. Metrika, 55:99-109.

Muller, H.-G. (1992). Change-points in nonparametric regression analysis. The Annals of Statistics, 20(2):737-761. 
Palangkaraya, A. and Yong, J. (2005). Effects of recent carrot-and-stick policy initiatives on private health insurance coverage in australia. Economic Record, 81(254):262-272.

Palangkaraya, A., Yong, J., Webster, E., and Dawkins, P. (2009). The income distributive implications of recent private health insurance policy reforms in australia. The European Journal of Health Economics, 10(2):135-148.

Qiu, P. (1991). Estimation of a kind of jump regression functions. Journal of Systems Science and Complexity, 4:1-13.

Qiu, P. (2003). A jump-preserving curve fitting procedure based on local piecewise-linear kernel estimation. Journal of Nonparametric Statistics, 15(4-5):437-453.

Qiu, P. (2005). Image Processing and Jump Regression Analysis. John Wiley \& Sons.

Qiu, P., Asano, C., and Li, X. (1991). Estimation of jump regression function. Bulletin of Informatics and Cybernetics, 24:197-212.

Qiu, P. and Kang, Y. (2015). Blind image deblurring using jump regression analysis. Statistica Sinica, 25:879-899.

Staudenmayer, J. and Ruppert, D. (2004). Local polynomial regression and simulationextrapolation. Journal of the Royal Statistical Society: Series B (Statistical Methodology), 66(1):17-30.

Stefanski, L. (2000). Measurement error models. Journal of the American Statistical Association, 95(452):1353-1358.

Stefanski, L. and Cook, J. (1995). Simulation-extrapolation: the measurement error jackknife. Journal of the American Statistical Association, 90(432):1247-1256.

Taupin, M.-L. (2001). Semi-parametric estimation in the nonlinear structural errors-invariables model. Annals of Statistics, 29(1):66-93. 
Wu, J. and Chu, C. (1993). Kernel-type estimators of jump points and values of a regression function. The Annals of Statistics, 21(3):1545-1566. 